\title{
Natural, Wearable Game Controllers
}

\author{
by Emily Steel
}

A thesis submitted to the Victoria University of Wellington in fulfilment of the requirements for the degree of Masters of Design Innovation

Victoria University of Wellington

School of Design, 2015 


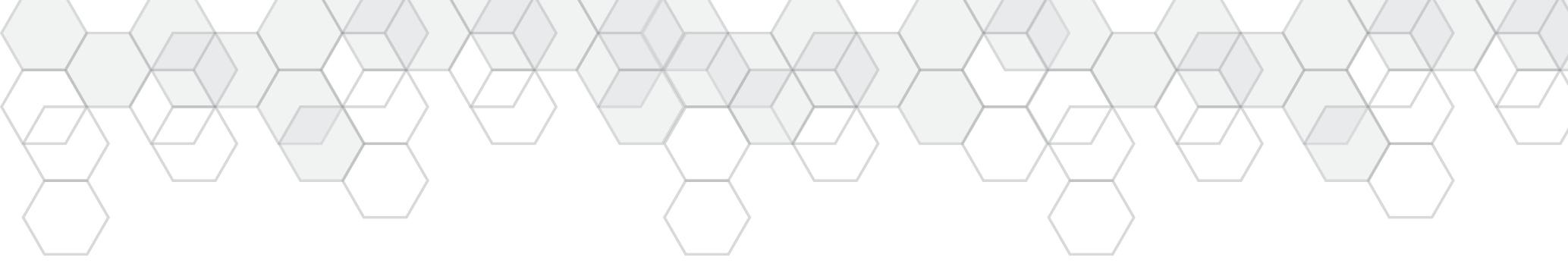




\section{Contents}

Acknowledgements

Abstract

1. Introduction

1.1 Thesis Overview 10

2. Gaming and Rehabilitation $\quad 12$

2.1. Abstract and Natural Game Controllers 15

2.2. In Home Gaming 19

2.3. Serious Gaming $\quad 20$

2.3.1. Nintendo Wii $\quad 21$

2.3.2 Microsoft Kinect $\quad 22$

2.4. Weight Bearing Asymmetry 23

3. Methodology 24

4. Hardware 28

4.1. Background 31

4.2. Sensor Development $\quad 32$

4.3. Sensor Mat Development 33

4.4. Ankle Brace Design 38

4.4.1. Brace Pattern 38

4.4.2. Sensor Placement and Construction 38

4.5. CNC Sewing Machine Sensor Construction 42

4.5.1. Pattern Experimentation 44

4.5.2 Fill Stitch Testing. $\quad 46$

4.6. Developing the Ankle Brace 48

4.6.1. Sensor Layout and Fabric Choice 48

4.6.2. Creating Structure with Stitching 51

4.6.3. Hexagonal Outline $\quad 52$

4.7. Wrap Band Development 54

4.7.1. Fixed Length Wrap Band 56

4.8. Electronics Development 60

4.8.1. Micro Controller and Wireless Technology 60

4.8.2. Battery Selection 60

4.8.3. Casing Development 63 
5.1. Integration with a Pre Existing Game 71

5.2. Exercise Assessment Tool 73

5.3. Custom Game For Hardware $\quad 75$

5.3.1. Initial Game Development 76

5.3.2. Further Game Development 78

5.3.3. Aesthetic Development 80

5.4. Integration with Hardware 83

5.4.1. Natural Interaction $\quad 85$

5.4.2. Addressing Weight Bearing Asymmetry 85

6. Conclusion 86

7. Discussion 90

References $\quad 92$

$\begin{array}{lr}\text { Appendix } & 96\end{array}$

$\begin{array}{ll}\text { 1. Sensors } & 97\end{array}$

1.1 Force Sensing Resistors $\quad 97$

1.2 Piezoelectric Based Sensing $\quad 97$

1.3 Velostat Based Pressure Sensors 97

2. Sensor Testing 98

2.1 Base Resistance Testing 98

2.2 Settling Time Evaluation $\quad 98$

2.3 Area Dependency 99

2.4 Fabric Dependency 99

3. Use of the CNC Sewing Machine 100

3.1 How the machine works 100

3.2 Conductive Thread as a Top Stitch $\quad 100$

4. Battery Selection 101 



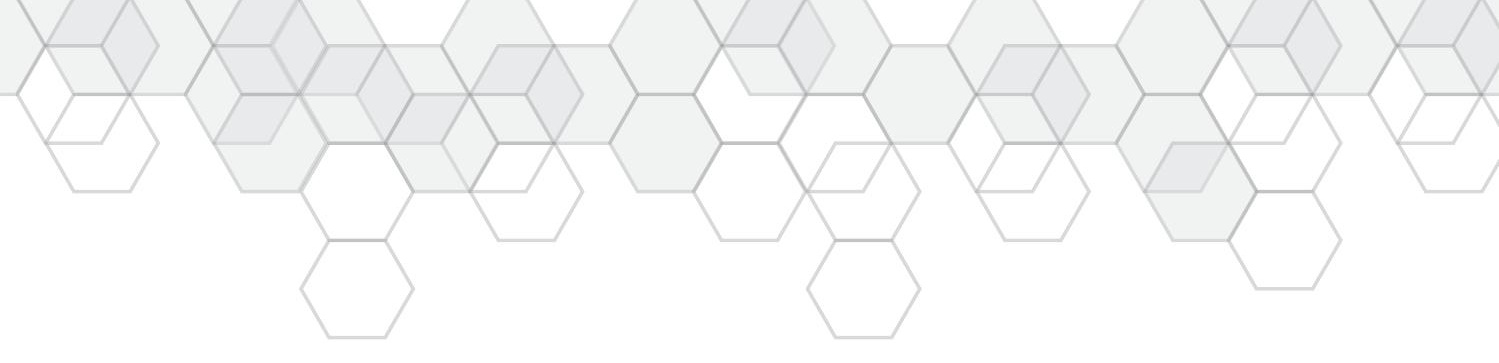

1. Introduction 

"How can wearable technology be used as a natural game controller to help with lower limb injury rehabilitation?" 


\subsection{Thesis Overview}

First the thesis will look at natural and abstract game controllers. The focus will be on defining the difference between the two and understanding how they have been introduced into a home gaming environment. It will then look at how these game controllers have been used in areas other than entertainment. The focus for this research will be on rehabilitation, specifically lower limb injuries. Chapter three will outline the methodology and methods that will be used during the design experiments.

The design experiments will be split into two sections:

\section{Hardware experiments}

2. Software / game design

The hardware design experiments will be conducted using an iterative design process. The first step will be to determine the technical requirements for the project. Once these have been established the aesthetics will be developed to accommodate the technical requirements of the design.

The software design experiments will focus on integrating the hardware with various applications and will be split into three parts:

1. Integrating the wearable with an existing game

2. Using the wearable as part of an exercise assessment tool

3. Creating a custom game focusing on exercise based movements

Subsequent chapters will discuss how successfully the hardware and software worked together. Finally the thesis will address any future uses and potential development for this project based on the conclusions made while conducting this research. 



In comparison to abstract controllers, natural controllers are either game or genre specific. There is also a spectrum in which these controllers fall into which spans how closely the controller emulates the real world action. These sorts of controller have been around since the early days of video games such as Gran Trak 10 (Atari, 1974) (Figure2.5), a single player arcade racing game featuring a steering wheel the player used to control the vehicle. A similar type of controller can still be found in modern gaming in a variety of driving games. This type controller is seen as a natural interaction because the interface has been mapped more closely to the action that would take place in the real world scenario(Skalski et al., 2011). Another example of a natural game controller is the Dance Dance Revolution (DDR) (Konami, 1998) (Figure 2.6) dance platform (arcade version) or dance mat (home console version). This is considered a natural controller because the users move their feet to trigger the character to dance. While the DDR controller falls into the natural category because the action of moving feet is closer to dancing than pressing buttons with fingers it is still not as natural as something like the car steering wheel. DDR actions are not the exact same as those performed by the user.

As well as falling into the spectrum of what can be considered a natural controller these controller can also be considered abstract if they are used to play another genre of game. The DDR controller could be used to play a game such a Pac-Man because the game play involves four buttons. However the DDR would not be a natural controller as the action of using feet does not coincide with the actions of the character. This will be an important distinction to keep in mind throughout the software development process of this thesis. The piece of wearable technology created during this project will have the opportunity to be a natural controller but the range of games it could be applied to is greater if it is allowed to be used as an abstract controller as well. 


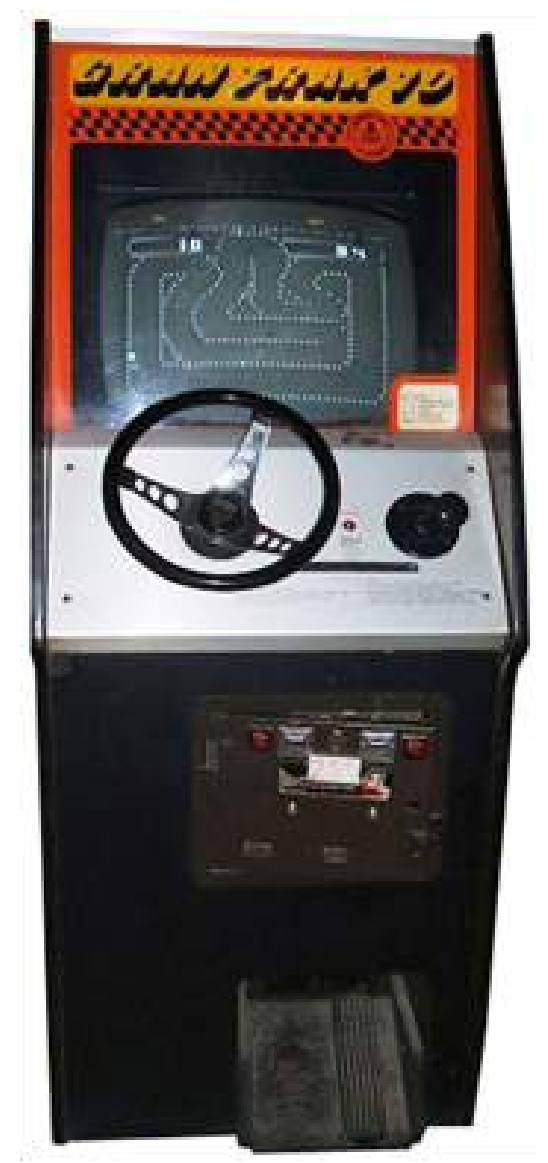

Figure 2.5 - Gran Trak 10 arcade machine (Atari, 1974)

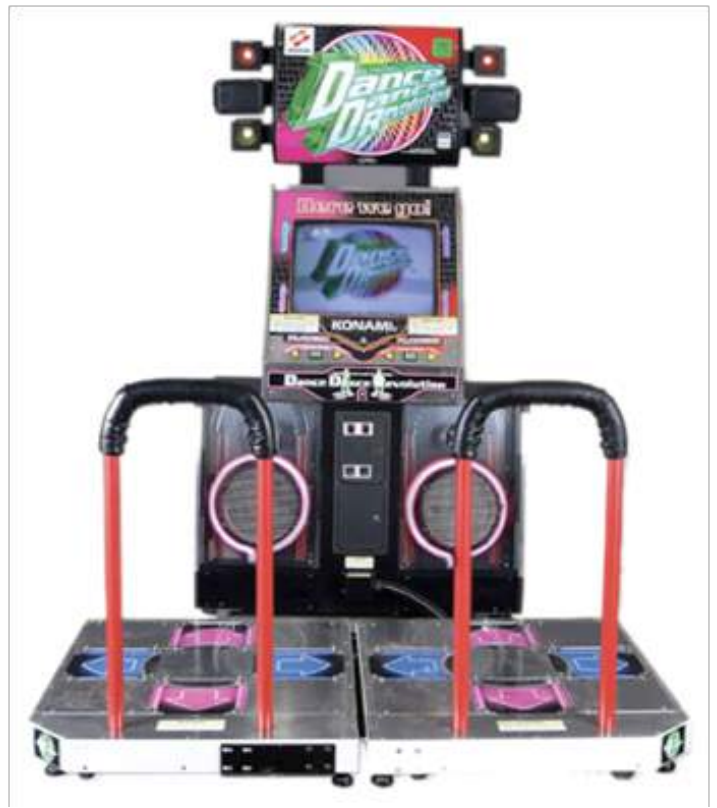

Figure 2.6 - Dance Dance Revolution arcade machine (Konami, 1998) 


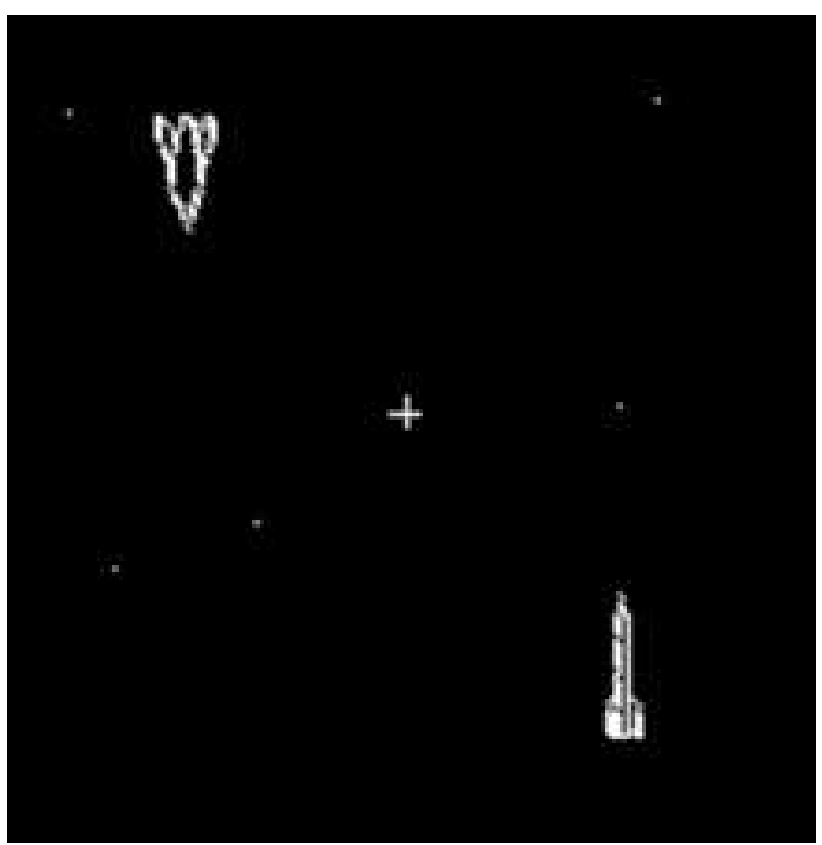

Figure 2.7 - Spacewar (Russell \& Graetz, 1962)

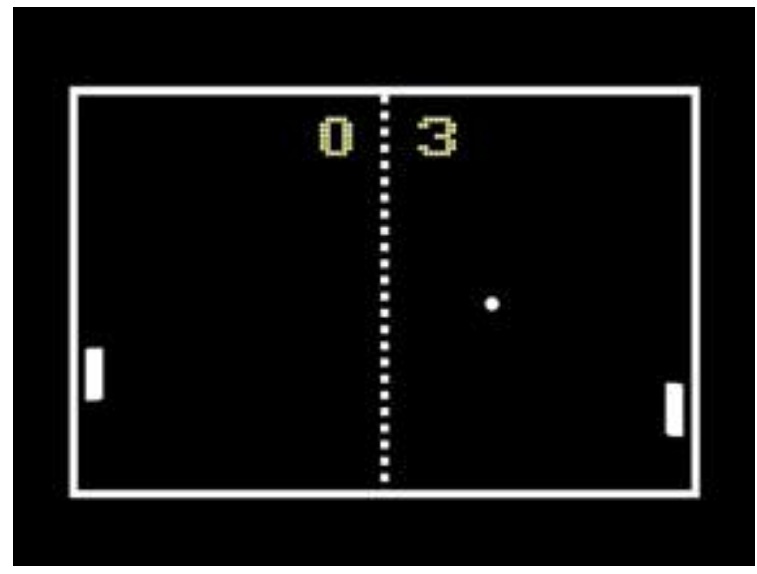

Figure 2.8 - PONG (Alcorn, 1972) 


\subsection{Serious Gaming}

From the earliest days of computing video games have been used for more than just entertainment. One of the earliest examples is the work conducted at the Rand Air Defence Lab in 1952 when the first military simulation games were created (Wolf, 2008). Since then people have looked at ways video games can be used to improve physical, emotional and cognitive responses (Kahlbaugh, Sperandio, Carlson, \& Hauselt, 2011 ). This section of the thesis will look at video game systems which have traditionally been used for entertainment and how they have been adapted to control serious games. Serious games are games which are used for more than entertainment. One of the main features of serious games is persuasion and influencing behaviour(Khaled, Barr, Biddle, Fischer, \& Noble, 2009). The focus of this thesis will be on serious games and their use in rehabilitation. With the emergence of gaming systems such as the Nintendo Wii and Kinect research is starting to be done into how these systems can be used for physical rehabilitation and injury prevention. An example of this is the use of the games in fall prevention and rehabilitation.

Falls account for approximately $34 \%$ of injuries in New Zealand annually, costing around $\$ 858$ million in 2013-2014 (versus $\$ 2.4$ billion in total claims cost) (ACC, 2013-2014). With such a high number of accidents being attributed to falls, prevention and cost effective rehabilitation are important areas to look at.. Several studies have been carried out looking at stand alone gaming rehabilitation systems, integrating these systems into pre existing programs and altering these systems so they can be used for things such as post stroke rehabilitation (see Bateni, 2012; Clark et al., 2010; Tanaka et al., 2012) 


\subsubsection{Nintendo Wii}

Several of the standalone Nintendo Wii programs were introduced and tested in retirement homes and care facilities. The focus was not necessarily on rehabilitation but aimed to increase the activity of the elderly (Kahlbaugh et al., 2011). During these studies it was found that the physical benefits from a Wii-based program were not conclusively better than traditional exercise programmes however there was a notable difference in emotional response. People participating in the Wii-based program reported better moods and were enjoying their exercise time more than people in traditional programs (Hsu et al., 2011). In one study it showed that the Wii provided residents in a retirement community with a new topic of conversation and the opportunity to form and maintain friendship based on a mutual activity

These findings are consistent with studies that show natural game controllers can have a positive effect on enjoyment during game play (Skalski et al., 2011). This improvement in mood has also been observed in using games played in an isolated setting and abstract controllers. In a study conducted with the elderly using the game Tetris it was found that participants who played the gamed for at least 25 hours showed improved moods. They used their new 'hobby' to form new friendships and it became a popular topic of conversation (Goldstein, 1997). Even though the social interaction did not take place during game play it is important to consider the effect of social interaction in rehabilitation. Whether the social interaction takes places during the exercise or it provides opportunities for social interaction after the exercise is completed. 


\subsubsection{Microsoft Kinect}

Another gaming system, which has been used for rehabilitation, is the Microsoft Kinect. An example of this is the Kinect-o-Therapy. The tool utilises the motion sensing capabilities of a Kinect and the interaction options this provides to create a simple, easy to use rehabilitation system (Roy, Soni, \& Dubey, 2013). Kinect-o-Therapy was tested with a range of physically impaired patients who were then questioned about the experience. Most people claimed the experience was enjoyable and preferred it to their traditional rehabilitation and would recommend it to other patients. However the study did not go in to analysing if the physical benefits of the system compared to traditional rehabilitation. The doctors who participated in the study responded positively to the system and contributed to the design development of the game (Roy et al., 2013). However there is no conclusive data stating the long-term benefit of using such a system for rehabilitation.

The use of the Kinect in rehabilitation reinforces the idea that fun and enjoyment are important for people going through the rehabilitation process. The Wii also reaffirms this idea by providing users the opportunity to engage in an activity that is both enjoyable and provides the opportunity to track progress through game play. One of the main issues with using games in rehabilitation is to make sure that the enjoyment does not diminish the physical benefits. While it is important for people to be able to track their progress and have fun, this should not be at the cost of a person's physical recovery. The existing literature suggests that while game based rehabilitation systems do have a place in recovery they should not completely replace traditional rehabilitation until they have been developed further (Kahlbaugh et al., 2011). 


\subsection{Weight Bearing Asymmetry}

Rehabilitation is a very broad topic. This thesis will focus on a specific problem within falls-related rehabilitation called WBA. WBA is when a person favours one leg over the other. In some people it can occur while standing still and can become more apparent while walking, running or exercising. It has been observed in people with lower limb injuries, neurological conditions, the elderly and the general population (Clark, Howells, Feller, Whitehead, \& Webster, 2014; Foo, Paterson, Williams, \& Clark, 2013; Jonsson, Henriksson, \& Hirschfeld, 2007; McGough, Paterson, Bradshaw, Bryant, \& Clark, 2012). Most people are unaware of this asymmetry even though it is a commonly occurring problem. WBA can lead to a lack of balance which can increase the likelihood of a fall especially in the elderly community (Jonsson et al., 2007)

Studies have been done with Nintendo Wii Balance Board (NWBB) to evaluate WBA. Previously, force-measuring platforms were used, however these were expensive, difficult to transport and required specific technical knowledge (Foo et al., 2013). The aim of these studies was to see if WBA could be reduced using NWBB. The NWBB was connected to custom software that provided the user with visual cues of their weight distribution. During these studies it was shown providing these visual cues helped to reduce WBA (McGough et al., 2012). 


Throughout the project a sequential mixed methods approach will be used to address the technical and aesthetic qualities of the proposed output. This approach is one in which "the researcher seeks to elaborate on or expand on the finding of one method using another method" (Creswell, 2011).

This strategy was chosen because the various components that need to be produced in this project need to be analysed in different ways. Using the mixed methods approach will allow the strengths of both qualitative and quantitative to be implemented (Kumar, 2014)

The project will start by using a quantitative approach to determine the properties of the sensors that will be the basis of the project. This will be used to show the relationship between the independent variables:
1. Base resistance
2. Area of velostat
3. Amount of conductive thread
4. Area contact between conductive thread and velostat
5. Material thickness

And the dependent variable:

\section{Output resistance}

The output resistance will be used to control the game so it is important to have a clear understanding of how the independent variables affect it. A quantitative analysis of the data is the best way to achieve this. This will be done using a scientific method where the variables are each isolated and tested multiple times to ensure consistent results. 
The next phase of the project will focus on implementing the sensors and determining how best to use them. Criteria for the hardware, a wearable and software, a game will be set out and then a series of design experiments will be conducted. After each experiment the iteration of will be analysed to see if it fits with the criteria listed below

\begin{tabular}{|c|c|}
\hline Hardware & Software \\
\hline 1. Comfort & 1. 'Natural' interaction \\
\hline 2. Appropriate placement of sensors & 2. Movement which addresses WBA \\
\hline 3. Ability to be put on and taken off & \\
\hline 4. Durability & \\
\hline 5. Ability to be washed & \\
\hline
\end{tabular}

This will be done using a qualitative approach where the success of an experiment will be determined by how well they met the criteria. This will be done by asking question about the design related to the criteria. The focus will be on identifying the problems that arose during the design experiment, why they occurred and how they can be fixed. A qualitative approach was chosen for this part of the project because it will be drive by these question being answered through subjective interpretation rather than empirical data.

The design experiments will involve the creation of several physical objects for the wearable in the form of a ankle brace and wrap band. During the creation of the wearable pieces experimentation with different manufacturing techniques will take place. The final design experiments conducted will be looking at software in the form of a pre-existing game, a Functional Movement System (FMS) (Cook, Burton, \& Hoogenboom, 2006) and the development of a custom game. During the experimentation process an active design approach method will be used.

A design will be created, analysed based on the criteria and then redesigned. The criteria will also be reviewed throughout the process to ensure it still aligns with the overall goal of the thesis. 




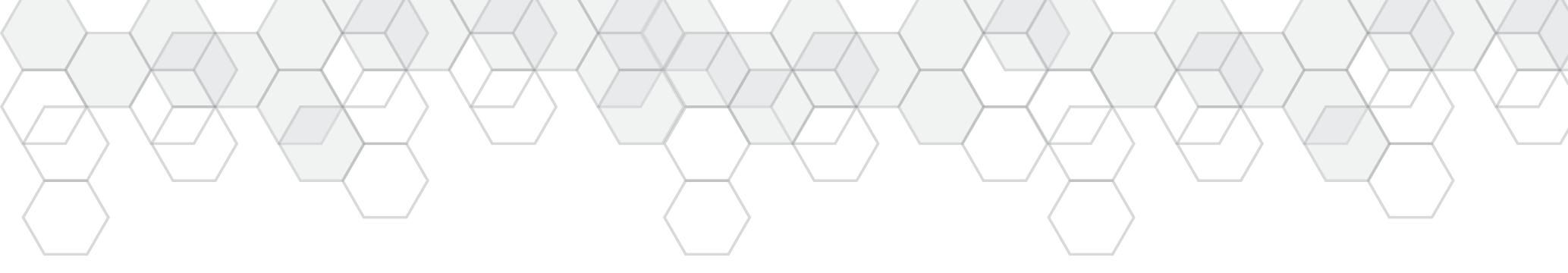




\subsection{Background}

The intention of the hardware part of the thesis was to create a piece of foot apparel that could be used to sense the pressure being applied to different parts of the users foot. For this reason the first thing to determine was which sensor would be best for the chosen application based on the following criteria:
1. Accuracy
2. Comfort
3. Cost effectiveness
4. Reliability
5. Able to be customised

Once a sensor was chosen the next step was to determine the exact properties of the sensor. Fully understanding the sensors would mean they could be used in the project more successful. Once sensor properties were determined the design created the best form for the wearable. During this process the following criteria were taken into consideration:

\section{Comfort}

2. Appropriate placement of sensors to measure pressure points

3. Ability to be put on and taken off

4. Durability

5. Ability to be washed

6. Aesthetics of the sensors

Three types of sensors were analysed. They were force sensing resistors, piezoelectric based sensors and velostat based pressure sensors. Appendix 1 further analysis of each type of sensor.

Velostat based pressure sensors were chosen because they are cost effective and easily able to be customised. The velostat sensors best align with the criteria set out. However there is limited literature surrounding exactly how they behave therefore. Individual testing would need to be done to determine their suitability prior to implementation. 


\subsection{Sensor Development}

For the intended purpose of this project velostat sensors were determined to be the best. The next step was constructing them and testing the effect of each property. Velostat sensors can be constructed to be any shape or size (Figure 4.1 and 4.2), but these different variables could potentially affect the sensor output. It was also important to look at the manufacturing process of the sensors to maintain consistency. Each sensor would need to include two layers of insulating material, velostat, conductive thread and a base resistor. For the insulating material neoprene was used because it had been proven to work in the creation of other pressure sensors (Perner-Wilson \& Buechley, 2010). The conductive thread chosen was made from spun stainless steel. Unlike most other conductive thread, which is made from coated nylon, spun stainless steel does not burn up when touched with a soldering iron. Once the general materials were selected a list of variables was created which could potentially affect the output of the sensor. These variables include:
1. Base resistance
2. Area of velostat
3. Amount of conductive thread
4. Area contact between conductive thread and velostat
5. Fabric thickness

Once the variables were determined sensor construction began. Appendix 2 provides individual notes on each variable. The sensor construction followed the same process for each test. The first step was to create digital laser cutting files for the sensors. Laser cutting instead of hand cutting was used to minimise human error in the sensor construction. The basic sensor shape was drawn out in Adobe Illustrator as a vector file and markings were added for the conductive thread-stitching pattern. Two pieces of neoprene and a single piece of velostat were needed for each sensor. Conductive thread was hand stitched through theses markings once the pieces were cut out. A piece of velostat was then put in between these two pieces that were then stitched together enclosing the velostat inside. 




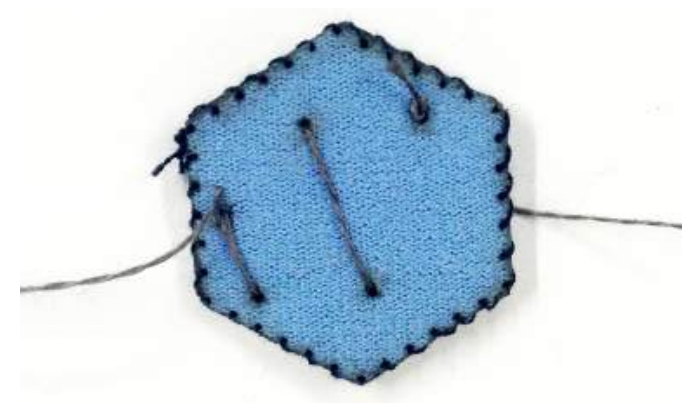

Figure 4.4 - Single hexagon sensor
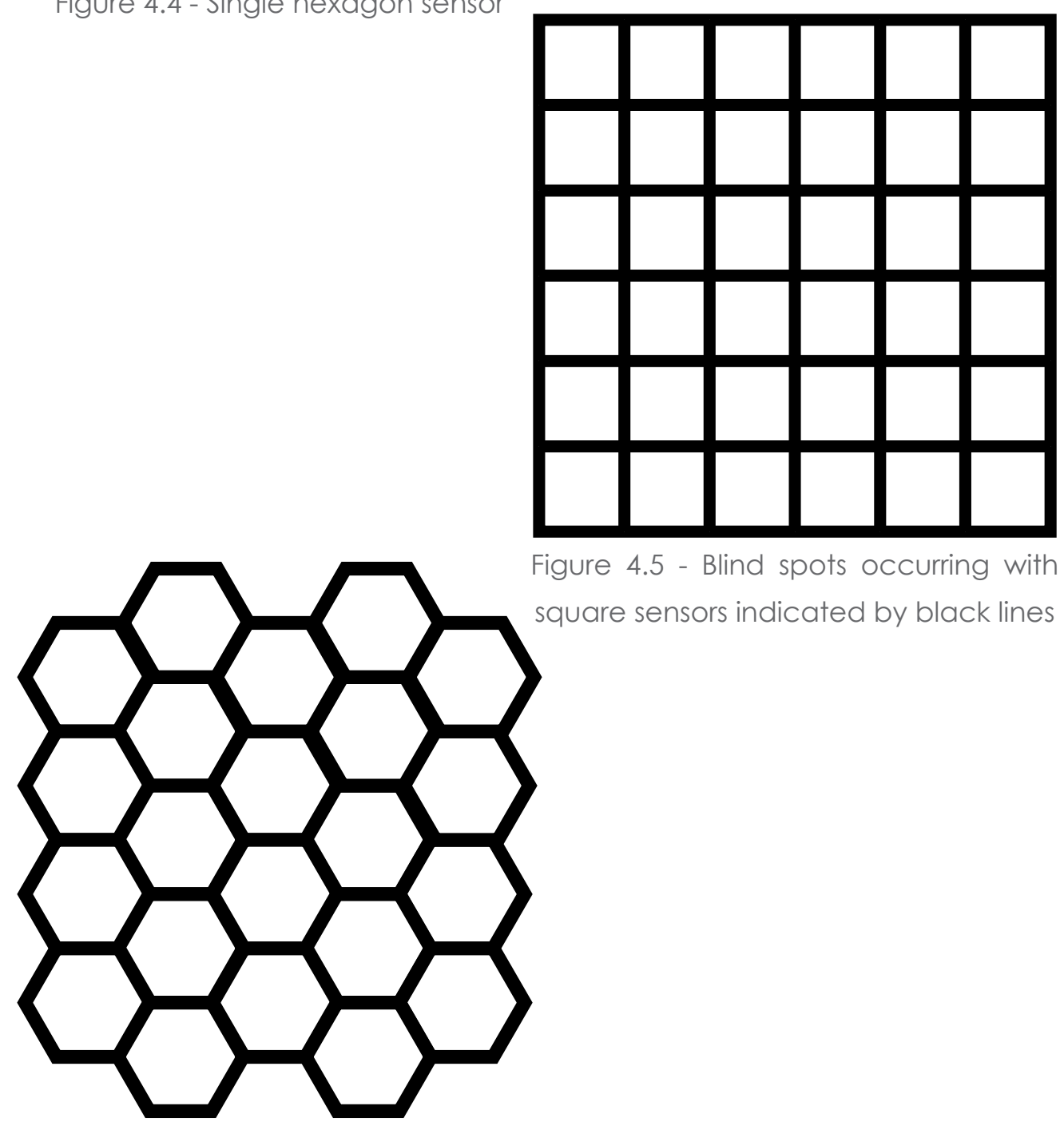

Figure 4.5 - Blind spots occurring with square sensors indicated by black lines

Figure 4.6 - Blind spots occurring with hexagon sensors indicated by black lines 
Using the hexagons allowed the same amount of surface area to be covered while increasing the sensitivity surface area. The mat was made using the same construction process as the individual sensors. However each sensor was marked out into a single piece of neoprene. The first mat was constructed by hand stitching each sensor and then each sensor was individually tested to confirm all were working using an Arduino matrix. Arduinos are open source microcontrollers which offer a simple way of interfacing hardware and software. In terms of this project they offer a way to measure the resistance of the sensors and communicate this data with the software/game. The particular Arduino used in this part of the project was an Arduino Uno. This model had six input pins meaning six sensors could be connected. A chip was used to create a matrix allowing all 27 sensors to be used at once.

After the first mat was constructed we decided to look into the possibility of using a sewing machine. This did not work on the individual sensors because of their small size however the larger surface area of the mat allowed for more control when sewing with a machine and would be able to reduced manufacturing time. During the construction of the second mat it was found that the conductive thread tracks could be sewn with a machine as long as the top stitch was standard cotton thread and the bottom stitch was the conductive thread. Being able to do this greatly reduced the manufacturing time and offered easier construction. Next the outlines of the sensors were stitched with cotton thread only however this proved unsuccessful for the same reasons as the individual sensors. The pieces of neoprene had the tendency to move around and catch in the velostat. This could potentially be avoided it we used an automatic industrial embroidery machine however we did not have access to one of these so proceeded with hand stitching the sensor outlines. Once the second mat was completed it was tested using the same set up as the first to see in the use of machine stitching had affected the properties of the sensors. There were slight differences between the two mats due to the different manufacturing techniques. To ensure consistency with the sensor each would have to be made using the same manufacturing technique. 
Once the second mat was constructed and tested and processing application was built to visualise the sensor data. The application was designed (Figure 4.7) so that when a hexagonal sensor was pressed on the mat the corresponding sensor would light up. The intensity of the colour would be dependent on how much pressure was being applied and multiple sensors could be pressed at the same time with different amount of pressure that would be shown by the visualisation. The mat was then integrated with another processing application in which a square would be moved around the screen based on which sensors where being pressed. For example if the sensors on the left hand side were pressed the square would move left and if the sensors of the right were pressed the square would move right. These two applications confirmed it was possible to control a computer-based visualisation with pressure sensors.

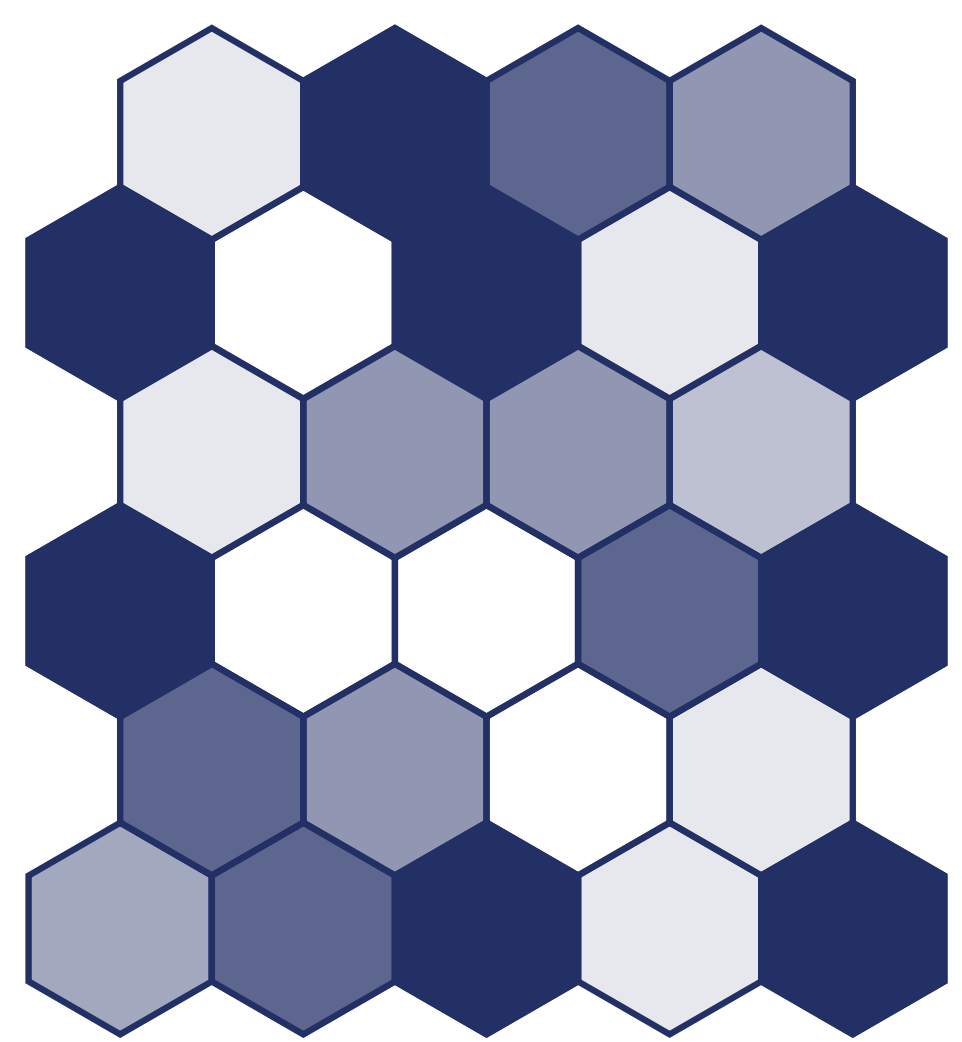

Figure 4.7 - Computer visualisation of pressure being applied to mat. Darker colour indicates more pressure 


\subsection{Ankle Brace Design}

An ankle brace was chosen as the appropriate article of clothing to begin prototyping with. It was chosen because it is already a piece of clothing associated with rehabilitation and is one which the public is familiar with. Criteria for the brace were determined based on the aims and objectives of this thesis. The ankle brace would have to fit the following criteria:
1. Comfortable and easy to wear
2. Reliable
3. Easy to manufacturing consistently
4. Cost effective
5. Washable
6. Aesthetically pleasing

\subsubsection{Brace Pattern}

The basic design of the ankle brace was modelled off an existing commercially available sports brace. The main areas of the foot that needed to be in contact with the brace were the ball and heel. The existing brace did not cover these parts therefore the pattern needed to be altered to make sure the right parts of the foot were making contact with the brace. The brace was placed on a foot and then fabric was added on the parts that need to be extended. This was then scanned and a digital pattern was as a vector file (Figure 4.8). The pattern was then cut out of a single piece of fabric and placed on a foot to see what alterations needed to be made. This process was repeated three times until the final pattern was determined.

\subsubsection{Sensor Placement and Construction}

Once the final pattern had been drawn up as a vector file, sensors were added to the parts of the foot that need to be measured. It was decided to not place the sensors under the arch of the foot because the pressure in this part did not need to be measured and it would greatly increase the number of sensors needed. This resulted in fourteen sensors covering the toe, ball, heel and side of the foot (Figure 4.9). 


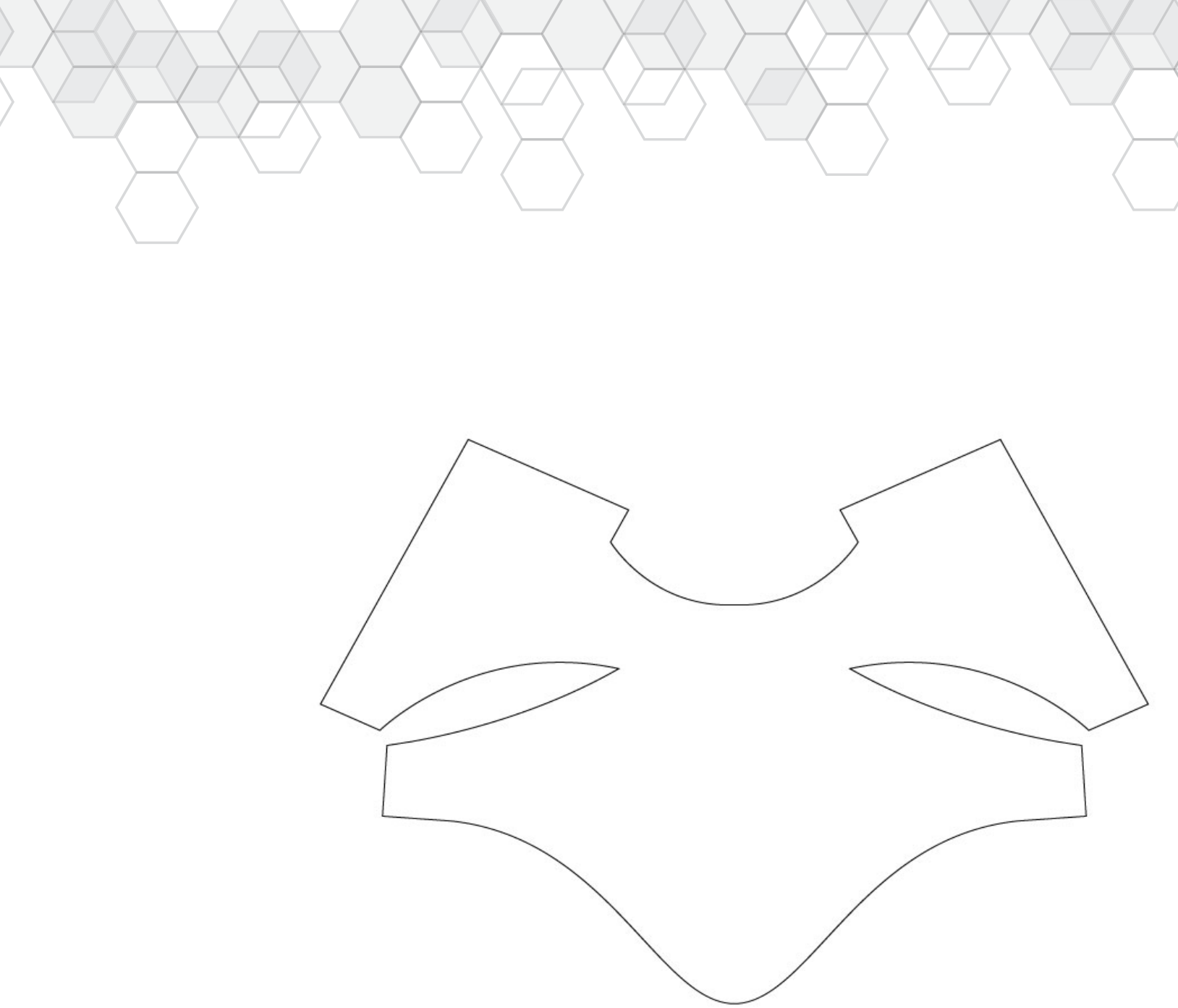

Figure 4.8 - Vector image of ankle brace pattern

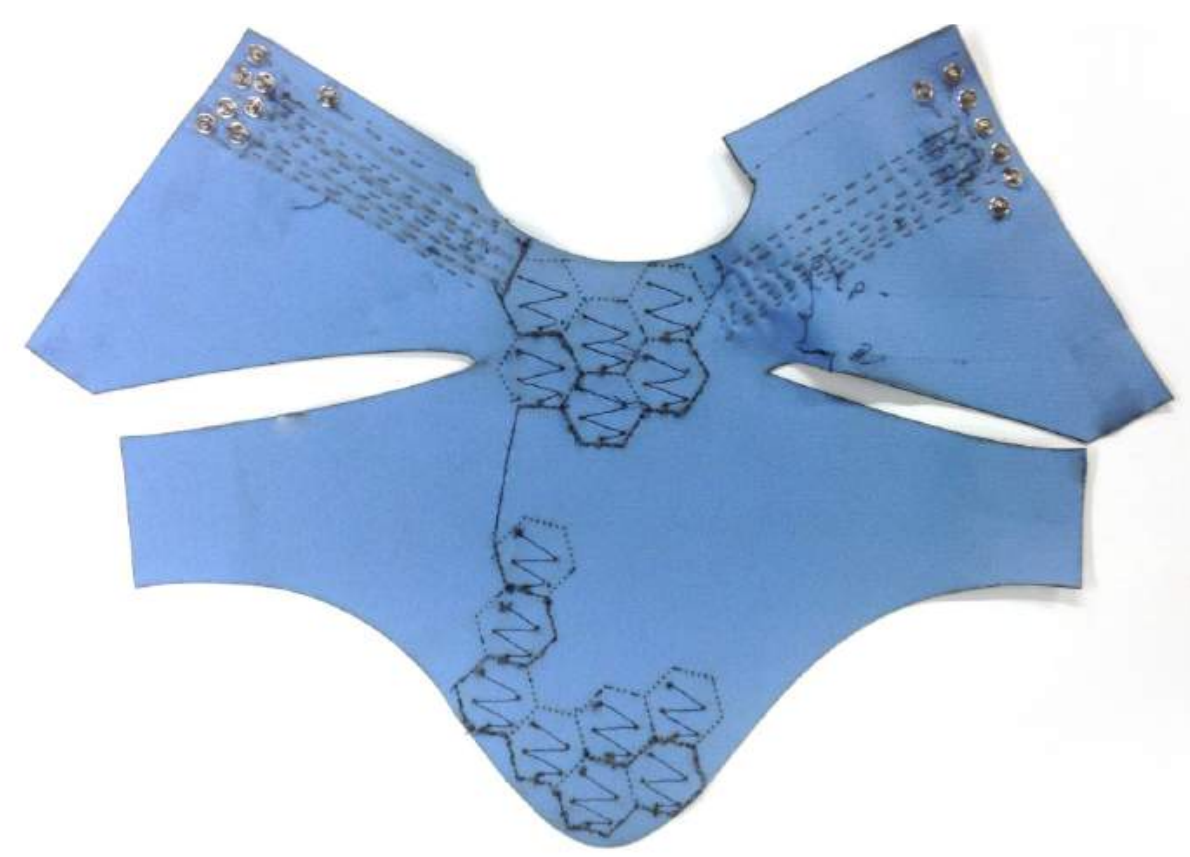

Figure 4.9 - Ankle brace with 14 sensors 
The brace construction followed the same process as the sensor mat. Once constructed the brace was attached to the Arduino Uno to test each sensor. Some of the problems encountered in the mat also appeared in the brace. The close proximity of the sensors to each other led to short circuits making several of the sensors couple. A new brace was created and tested through each stage of the manufacturing process to see at which point the coupling started to happen. During this process two points during the construction appeared to be causing the issue. Conductive thread touching the velostat in two sensors at the same time and conductive thread fraying and connecting two of the tracks.

The first issue was resolved by securing the conductive thread better and ensuring it was fully cover by the a single piece of velostat. To do this a small piece of double sided tape was placed on the neoprene and then the velostat was stuck on to this making sure no conductive thread was sticking out the side before the other piece of neoprene was secured on top.

To resolve the next issue a layer of insulating fabric was added. Conductive fabric tracks were sewn into a piece of cotton and then covered with another piece of cotton on either side. On each side of the conductive thread nonconductive thread was used to isolate the threads from each other in the attempt to prevent short circuits. Even using this technique the thread would sometimes fray and small parts from one track would touch another track. The next attempt to solve this problem was by using liquid insulation tape. A track was sewn and then covered in the liquid insulation tape. Once the tape had dried another track was sewn. In some of the prototypes this techniques was able to eliminate the coupling but in other it did not. As each brace took several hours to construct before it could be tested and then did not always work it was decided that this technique did not meet the manufacturing criteria that had been determined. Having more sensors would provide more data however this advantage was outweighed by the difficulty of creating reliable braces with this many sensors. 



\subsection{CNC Sewing Machine Sensor Construction}

Experiments were connected to determine if a CNC sewing machine could be used for production. The main issue was to see if conductive thread could be used in the machine. After it was determined that this was a feasible method of construction, a small series of samples were completed using a variety of stitching pattern with conductive and non-conductive thread. . For more information of how the machine works and thread testing see Appendix 3. Neoprene was the fabric choice for previous sensors and was used in the initial samples. Cotton samples were also created to see if a different fabric could be used to increase the design options later on. Figure 4.11 shows a working pressure sensor that was created based on what was learned during a series of tests. Figure 4.12. to 4.19 detail the various stitching combinations and the outcomes of each test.

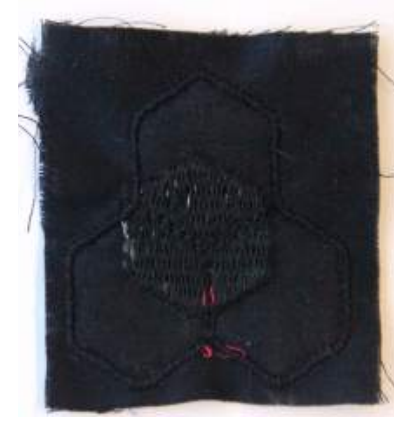

Figure 4.11

- Cotton fabric

- Conductive base stitch

- Non-conductive top stitch

- No overlay stitch

- Non-conductive outer stitch

- Two layers with a velostat centre

- Working pressure sensor
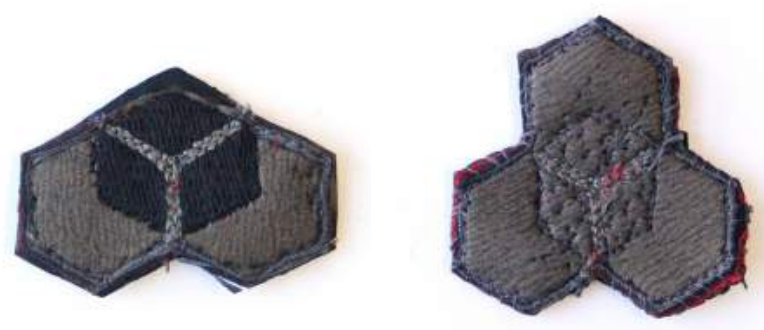

Figure 4.12

- Neoprene fabric

- Non-conductive base stitch

- Non-conductive top stitch

- Conductive overlay

- Non-conductive outer stitch

- Two layers with a velostat centre
Figure 4.13

- Neoprene fabric

- Non-conductive base stitch

- No top stitch

- Conductive overlay stitch

- Non-conductive outer stitch

- Two layers with a velostat centre 



\subsubsection{Pattern Experimentation}

The samples confirmed the CNC sewing machine could be used to create functional pressure sensors. After making this discover it was then decided to see the types of patterns that could be created using the sewing machine. This would allow the creation of more interesting designs while keeping them functional. The first test done was using a simple hexagonal pattern outline with no fill (Figure 4.20) followed by a similar pattern with fills and conductive stitching (Figure 4.21)

During the creation of Figure 4.21 the thread being used ran out and was replaced with another colour. In the hexagon where the thread ran out the effect of mixing the two colours makes the layering of stitches more apparent and creates quite an interesting aesthetic. During the sewing of the conductive overlay the thread kept breaking. This was sometimes due to the imperfections in the thread mentioned earlier. The other cause of the thread breaking was the thread running over itself. Due the thickness and material of the thread when it was sewn over itself it tended to catch and break. This is not an issue for small amounts of stitching as demonstrated by previous samples but could become a problem later on especially as having to re-thread the machine regularly does become time consuming. The solution would be to create conductive thread patterns that never cross over themselves. While this may add more time to the pattern design process it will reduce manufacturing time and increase reliability. The more conductive thread used also increases the resistance so making sure the thread does not run over itself will decrease the amount of overall resistance. 



\subsubsection{Fill Stitch Testing.}

When filling in a shape the embroidery machine offers several options as to what kind of stitch can be used. All previous tests used basic weave stitch. A weave stitch is a layer of stitching which is then overlaid with more stitches. The stitch length and width determine the type of weave that is created. Other types of fill stitches include stain, motif and embossed. 16 samples were created, the majority of which used non-conductive thread (Figures 4. 22 -4.37) and then four of the fills were repeated using conductive thread. Two of the samples failed (Figure 4.34 and 4.35), one sample was incomplete (Figure 4.37) due to the thread breaking and one sample was successful (Figure 4.36). The caption on the figures are what the fill stitch was set to create the hexagon.

These samples confirmed there are limitations to the complexity of patterns that can be sewn using conductive thread. In spite of this conductive thread can still be used in combination with non-conductive thread to make more interesting patterns and circuit layouts than hand sewing. The different fill stitches also offer the opportunity to alter the stretch of the fabric. Fill stitches with a high density such as the weave stitch constrain the stretch of the fabric and give it more structure. Stitches with a low density reduce the stretch but leave the fabric with the ability to bend and move. 


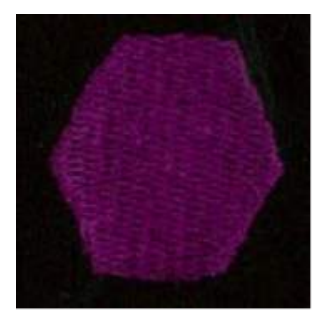

Figure 4.22 Weave (1)

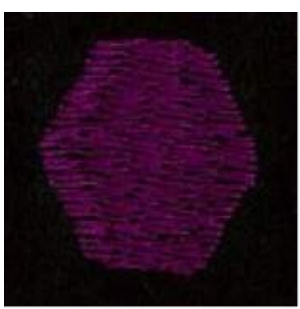

Figure 4.26

Satin (50\%)

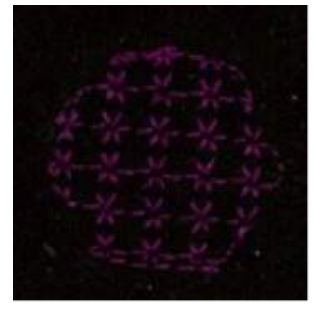

Figure 4.30 Motif (Star07)

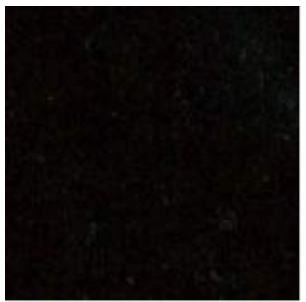

Figure 4.34 Repeat of 4.30

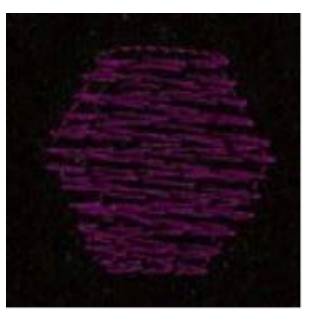

Figure 4.23

Satin (20\%)

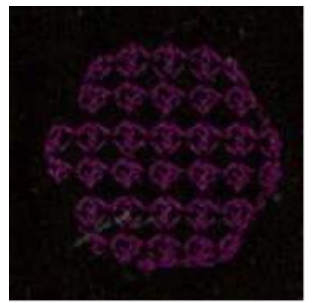

Figure 4.27

Motif (M6)

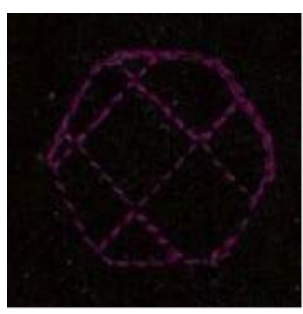

Figure 4.31

Motif (BW-Kite 1)

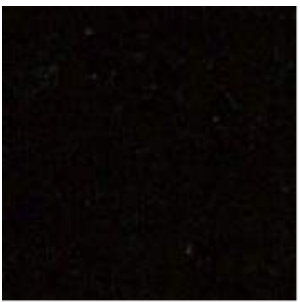

Figure 4.35

Repeat of 4.3

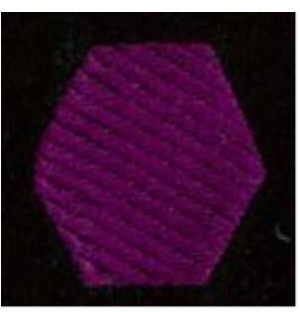

Figure 4.24

Weave (4)

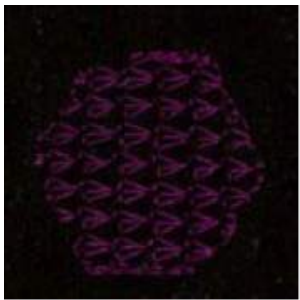

Figure 4.28

Motif (M14)

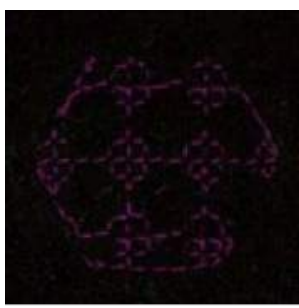

Figure 4.32

Motif (BW-Cross8

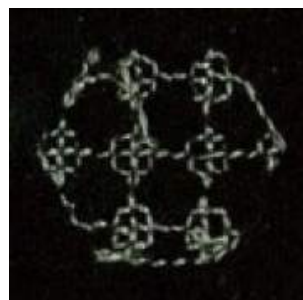

Figure 4.36

Repeat of 4.32

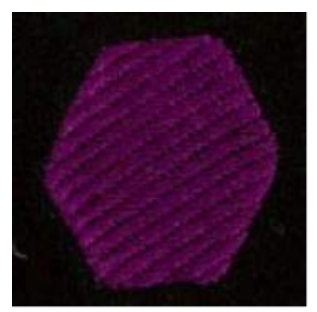

Figure 4.25

Weave (15)

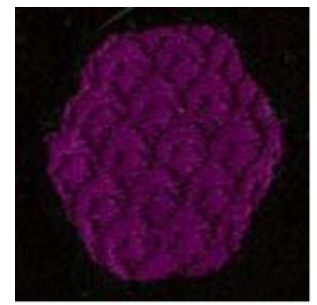

Figure 4.29

Embossed (S1)

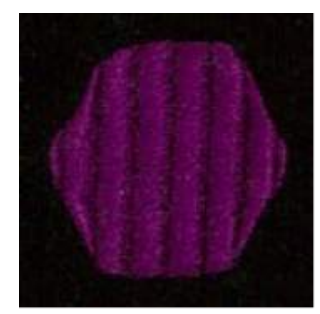

Figure 4.33

Embossed (W1)

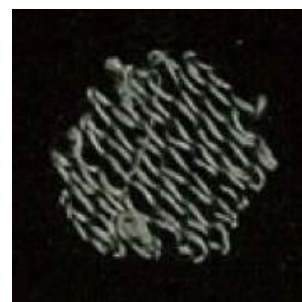

Figure 4.37

Repeat of 4.33 


\subsection{Developing the Ankle Brace}

\subsubsection{Sensor Layout and Fabric Choice}

The basic ankle brace pattern and sensor layout was developed during the construction of the handmade version. Using the initial layout the number of sensor was reduced from four to five. The shape the design was then altered to take advantage of the CNC sewing machines abilities. A layout was created in Illustrator using the pre-determined sensor layout and incorporating the hexagonal pattern tested in previous samples (Figure 4.10). This layout was then converted to a stitching file and sewn on to merino. Merino was used instead of cotton because the ankle brace needs to stretch which cotton does not allow. Using merino did present some challenges because of its lightweight nature and stretch. When sewing the machine would stretch the fabric which would often result in messy misaligned outcome. Even when using embroidery support material and altering the machine settings for stretch fabric the problem still occurred. To combat this issue lightweight interfacing was fused onto the fabric before it was place in the embroidery hoop. The interfacing gave the fabric more structure and reduced the stretch allowing it to be sewn with more ease. The interfacing could then be removed after sewing returning the fabric to its original stretchiness.

Once the issues with the fabric had been resolved the pattern could be sewn. Conductive thread was used for the sensors and non-conductive was used for the rest of the pattern. The first sample created (Figure 4.38) used a single line of conductive thread covered with a satin stitch of non-conductive. Due to the stretch of the fabric the non-conductive overlay was not as successful as it had been in the cotton fabric samples. The stitches misaligned and it was still possible to see the conductive thread sticking out from underneath the non-conductive. The next sample (Figure 4.39) did not include the satin stitch overlay on the conductive thread. The conductive thread was sewn first and then the non-conductive thread pattern. Each conductive thread track was then tested with a multi-meter so see if it could successfully carry a current from the sensor start point to the end of the track. 


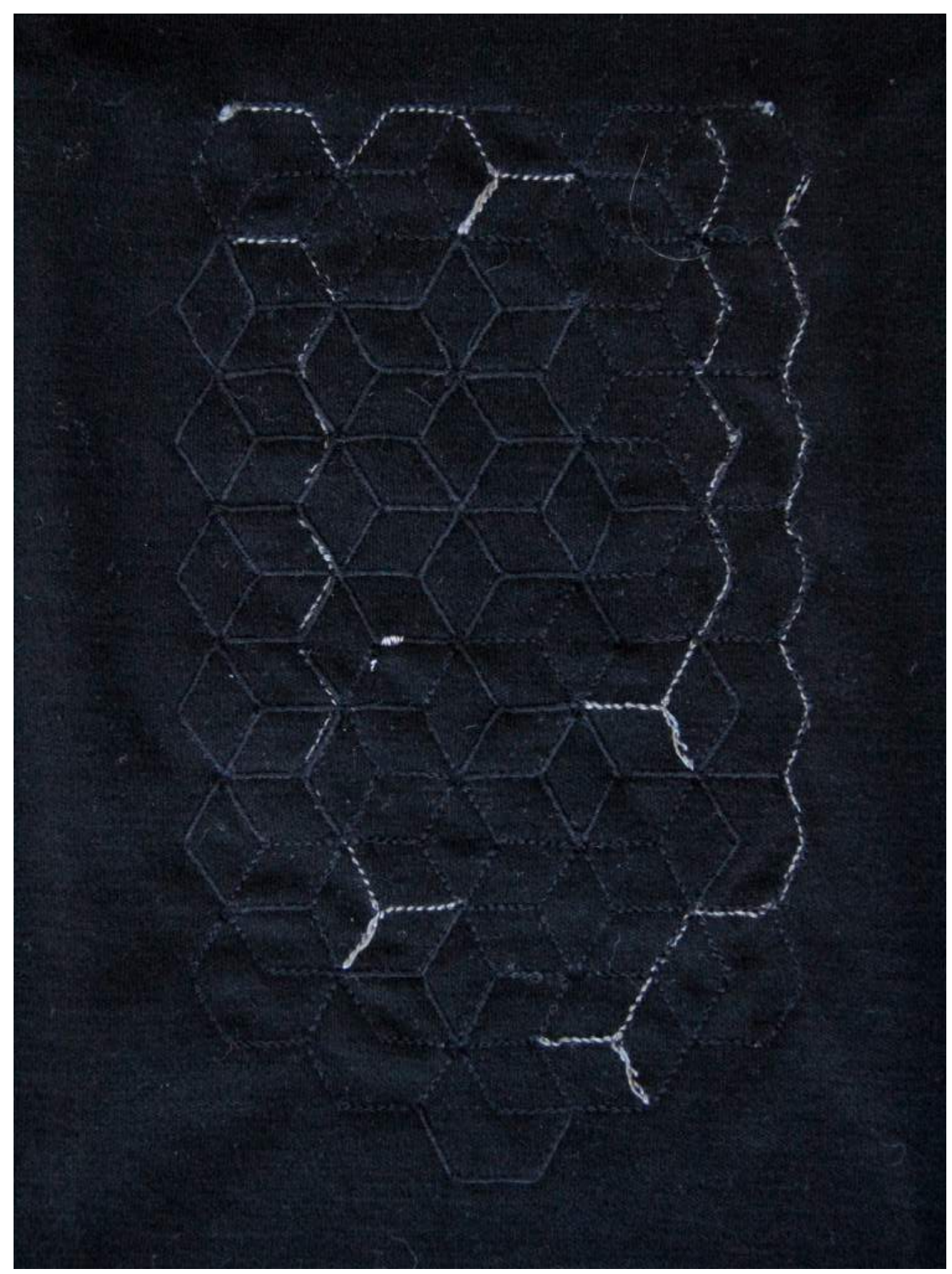

Figure 4.38 - Hexagon pattern with conductive tracks covered in satin stitch 



\subsubsection{Hexagonal Outline}

All previous interactions of the brace were modelled off the same pattern using a combination of curves and straight lines. As the hexagonal pattern is being used as both a structural and aesthetic component in the stitching pattern it was decided to see if it was possible to bring this motif into the outline of the of the brace design. Hexagons were laid out within the original pattern trying to get them as close to the original outline as possible. The main focus of this small was to see what the front part would look like. Once the hexagon pattern was laid out it was converted to a stitching file using a combination of stitch. Sensors used a stain fill, conductive tracks used a single run stitch and the rest of the pattern used a satin line stitch.

The brace was stitched at a scaled down size (Figure 4.42 and 4.43) to get an idea of the overall shape before making a full sized one. During the sewing process the black thread ran out and the sample was removed from the embroidery hoop prior to the design being completed. The front section was complete and as this was the main focus of the sample a new one was not made.

When the brace was wrapped up in the way it would be on a foot the front section did not fold up nicely and looked awkward. A potential way to solve this would be to extend the front section of the brace and fold it up so it formed a closed toe similar to a ballet shoe. A rough outline of a full sized brace was created and then placed on a foot. Fabric was then attached to the existing brace and modelled to create a toe cover. The brace was then removed and cut into a flat shape so it could be scanned and turned into a digital pattern. The new brace was then stitched in a scaled down size and sewn together to get an idea of the shape.

Two iterations of the scaled down brace were made each time adjusting the shape of the toe section. Adjustments were made so the hexagons lined up with each other and there was minimal overlap. Once the pattern was finalised 


\subsection{Wrap Band Development}

After a review of the ankle brace it was decided that a the wearable would take a new form. To do this inspiration was taken from injury apparel and after sketching (Figure 4.44) it was decided the new form would be a wrap band. The wrap band would be a long piece of material that would wrap from the toe around the foot to the heel and secured in place on the ankle. The different variations of the wrap-band based ankle brace would be explored:

1. Customisable sensor placement

2. Stretchable

3. Fixed length and sensor placement
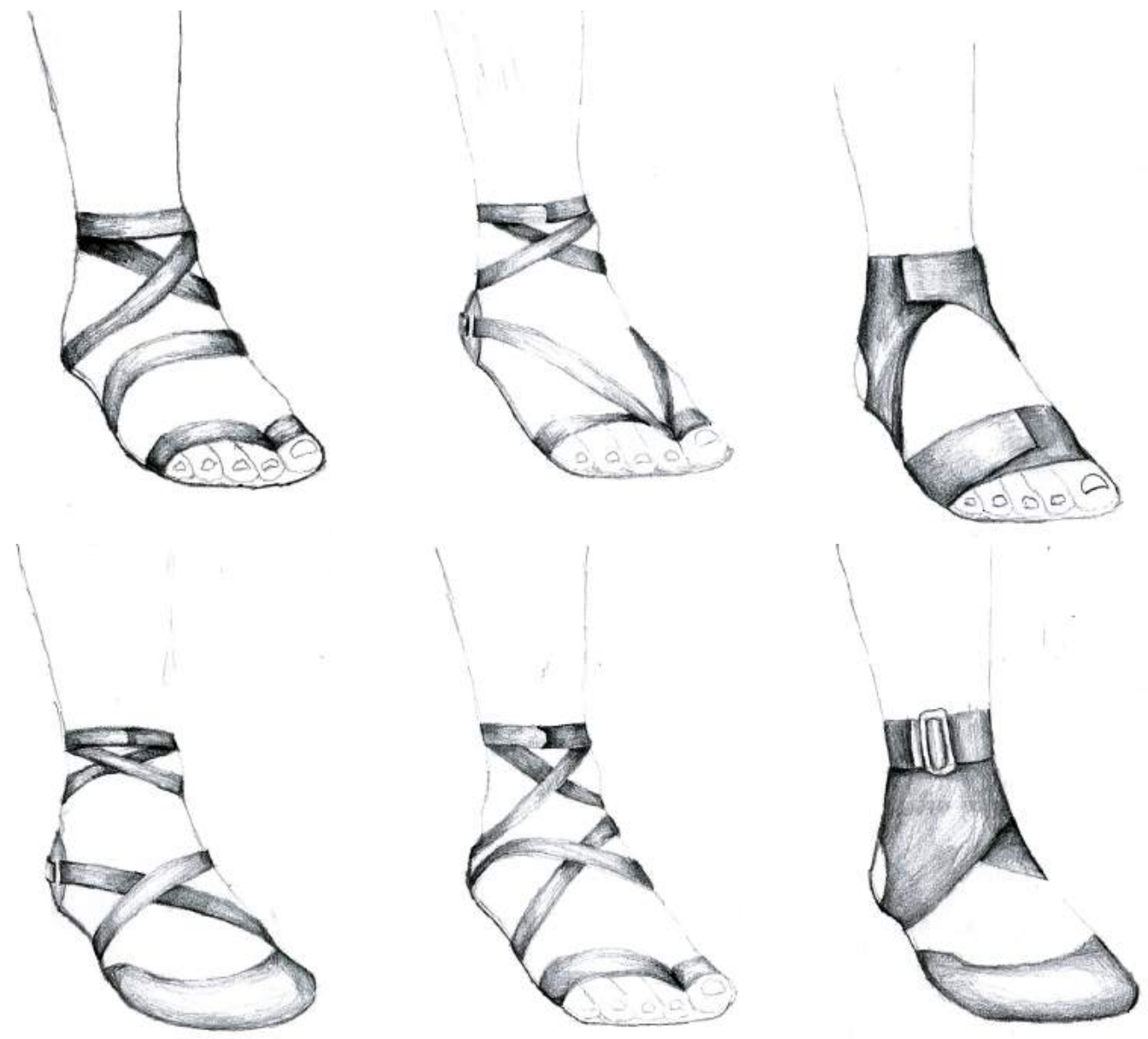

Figure 4.44 -Sketches of foot apparel designs 


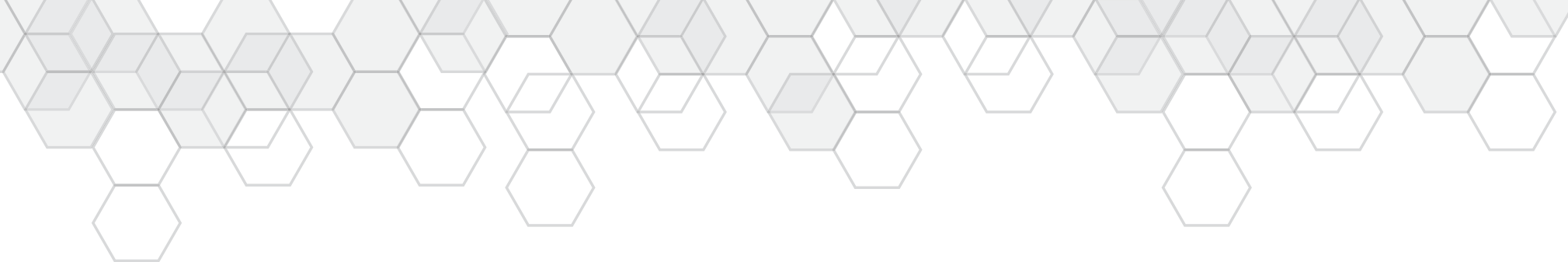

The first wrap band explored was one with customisable sensor placement. The user would be provided with a wrap band, detachable electronics, four sensor and instructions on how to attach them. This would mean that one kit set would be created and would be applicable to any sized foot. The main problem with this is this tracks would be have to be created along the full length of the band and would have to be exposed to the user would know where to attach them. Having the track exposed meant that they could not be properly insulated. Short circuits could be created when the user is attaching which could damage the whole band..

Then next option to be explored was the creation of a stretchable band which used stitching to create structure. This did not work in the ankle brace because it had two different directions of stretch which distorted the shape of the hexagons. In the wrap band stretch would only happen in one direction which would decrease the amount of distortion. The main problem with a stretchable wrap band is the conductive tracks because the thread used to create them has no stretch. If the conductive thread is sewn in a straight line it would reduce the amount of stretch the fabric could have. Over stretching the band could also lead to the thread breaking. A way to address this was to sew the thread using a zig-zag stitch. This is the stitch usually used when sewing with stretch fabric.. To make sure that the zig-zag's were wide enough so they did not touch each other, the wrap band would have to be made wider than would comfortable around some parts of the foot.

The final design explored for the wrap band was the fixed length option. Sensor position and band length were determined before manufacture started. These two properties would be altered depending on what measurements were required for the particular foot size. The band would come in different size ranges for example a person with a size 6 foot would be able to wear the same band as a person with a size 8 foot depending on foot width. The band would also work for either the left or right foot so only one pattern would need to created. 


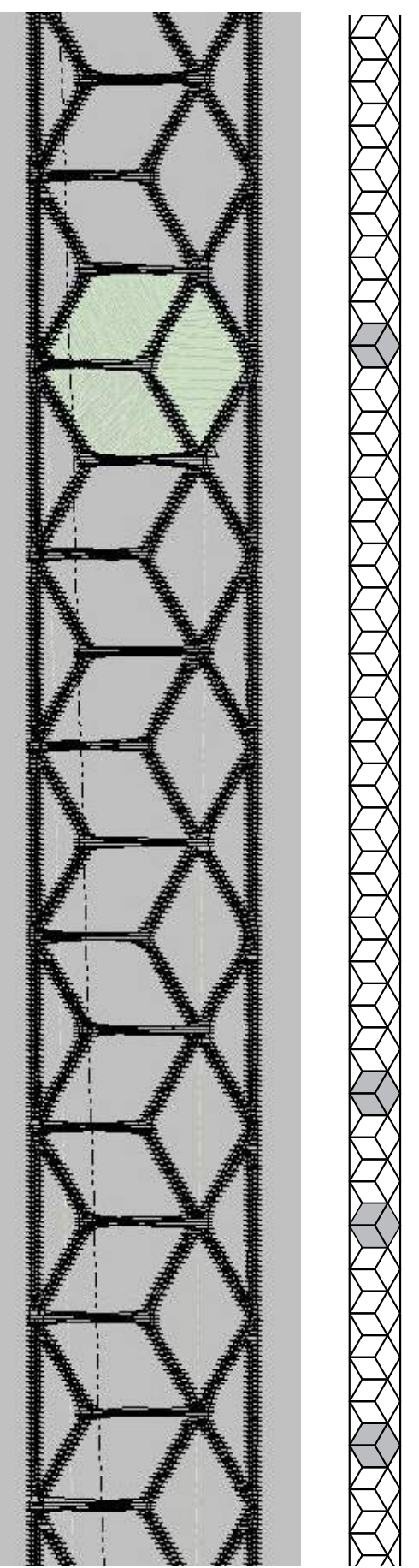

A new wrap band was created with the stitching pattern extending the length of the band (Figure 4.46 and 4.47). It was given a rectangular outline to minimise the manufacturing time as a hexagonal outline would add time to the construction process later on. Once the top layer was sewn the sensors were completed and conductive thread was sewn from each sensor to the end of the band. The fabric was then folded to insulate the conductive tracks. The band was then wrapped around the foot to see how it looked and felt. It was decided that the band was to wide (Figure 4.48) and was wrapping around the foot too many times creating unnecessary bulk.

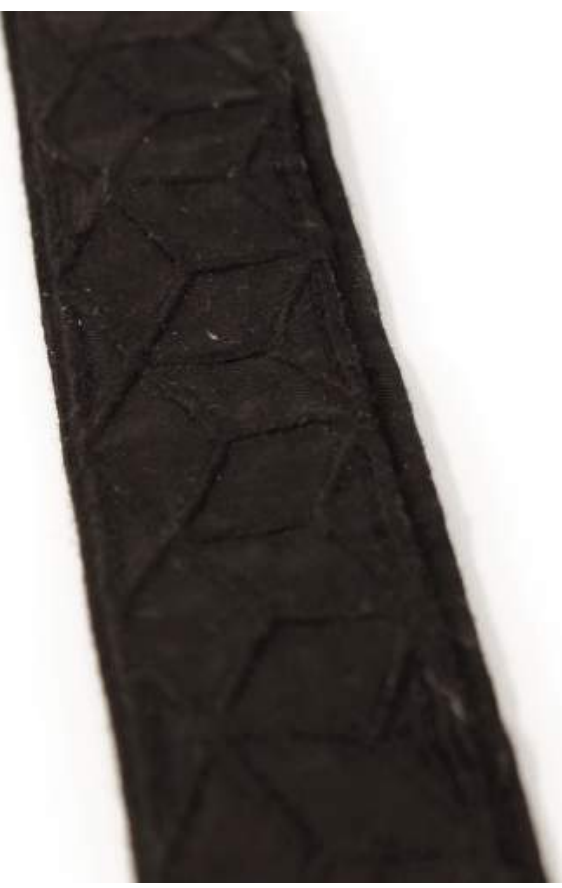

Figure 4.46 - Close up of band stitching file

Figure 4.47 - Vector file of full length band

Figure 4.48 - Close up of band 
A new pattern was created adjusting the position of the sensor so the band would wrap around the foot less. To adjust the band width the sensor could be made smaller however making them smaller would also make it harder for them to be touching the right parts of the foot for different sized feet. A new pattern with a hexagonal outline and altered sensor position was created(Figure 4.49 and 4.50) Even though the hexagonal edge adds time to the manufacturing process it reduces the bulk of the width without having to reduce the sensor size. the hexagonal edge is also more aesthetically interesting and emphasises the stitching detail (Figure 4.51)

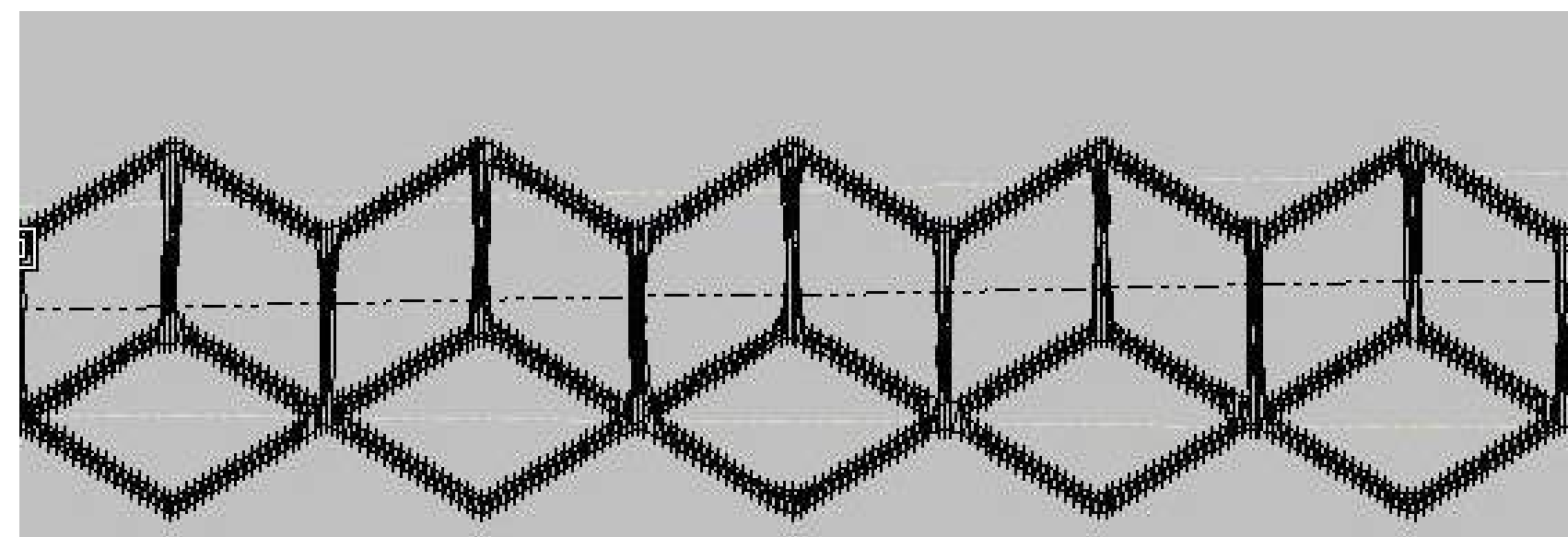

Figure 4.49 - Close up of hexagonal band stitching file

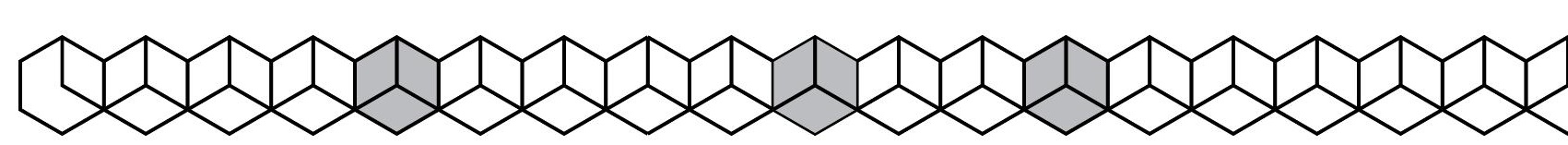

Figure 4.50 - Vector file of full length band 


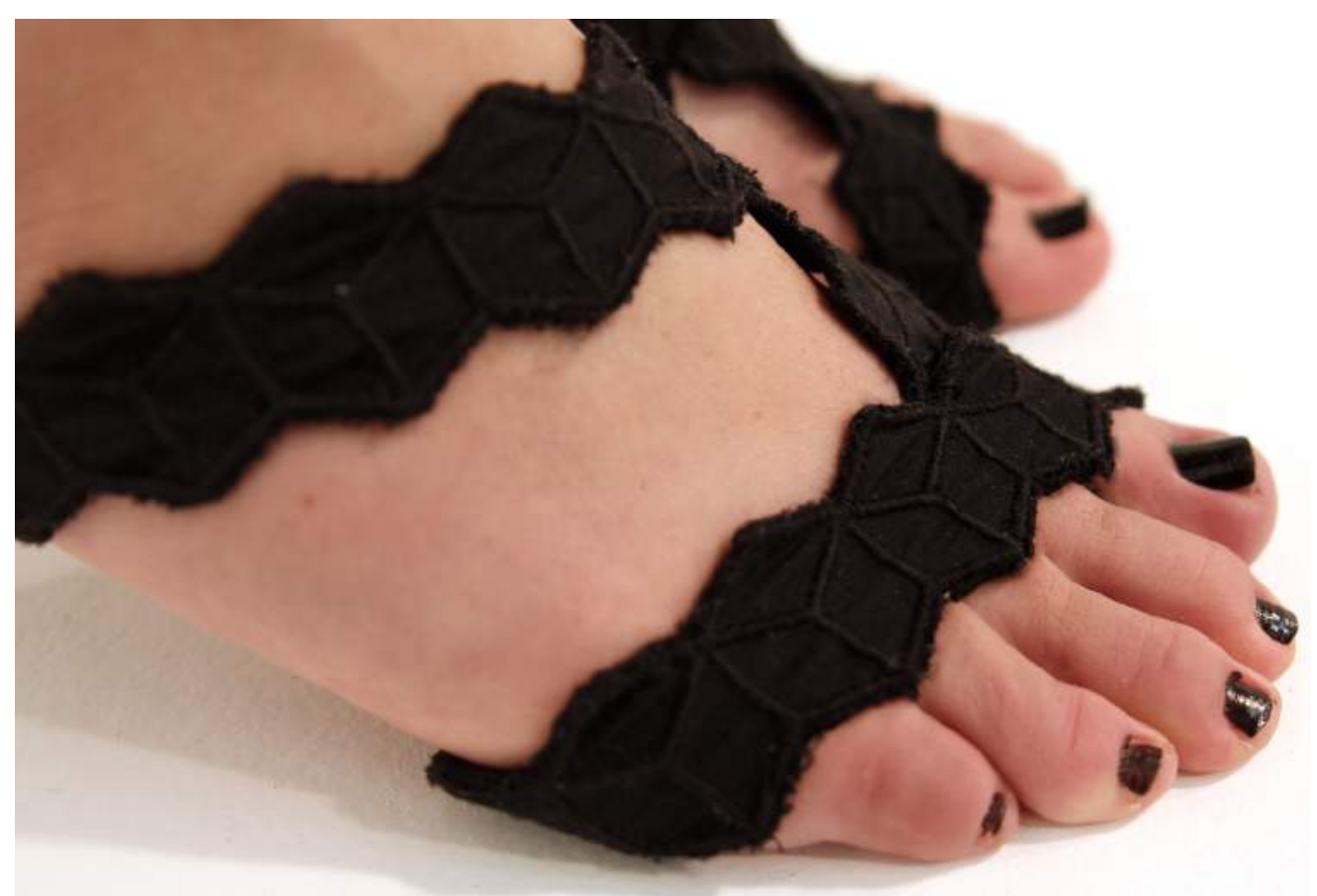

Figure 4.51 - Close up hexagon stitching detail

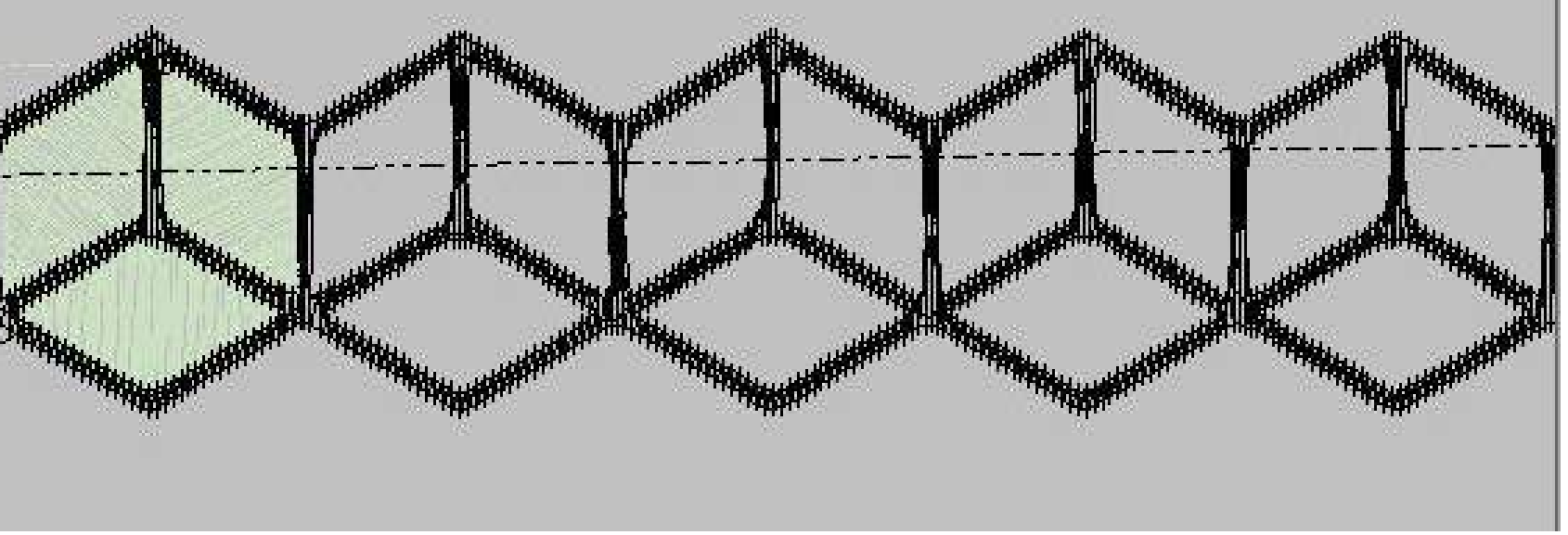

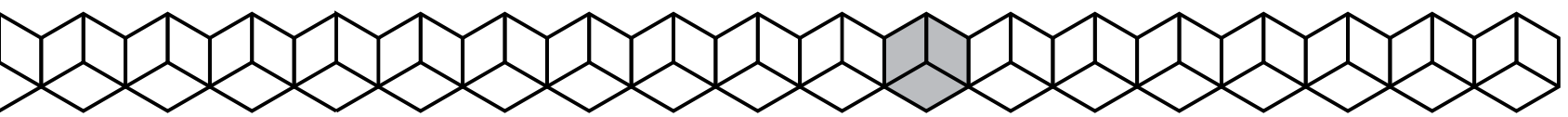




\subsection{Electronics Development}

\subsubsection{Micro Controller and Wireless Technology}

In the sensor experiments an Arduino Uno was used to interface with the computer. The Uno was mainly chosen because it lends itself to prototyping. This made it easy to test a variety of sensors without having to spend time soldering circuits. However for the wrap band a different type of micro processer and electronic set up would be required to fulfil the following criteria:
1. Removable
2. Wireless
3. Unobtrusive
4. Rechargeable

Based on the criteria an Arduino Pro Mini (Figure 4.52) was chosen. The Pro Mini is considerably smaller than an Uno while still providing the same functionality. To make the design wireless a Bluetooth Mate Silver chip (Figure 4.53) was connected to the Arduino allowing it to connect to the computer while not restricting the user's ability to move around.

\subsubsection{Battery Selection}

To keep the brace portable batteries would need to be added to the circuit so it could be powered independently from the computer. The following battery solutions were considered (Figures $4.54-4.56$ ):
1. Standard AA
2. Coin cell
3. Polymer Lithium Ion (LiPo)

Each battery option was analysed to see how it best fitted the criteria of the electronic setup. For more information see Appendix 4. 


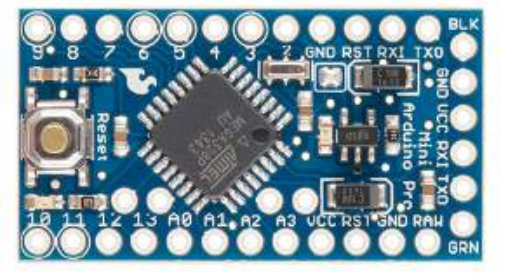

Figure 4.52 - Arduino Pro Mini

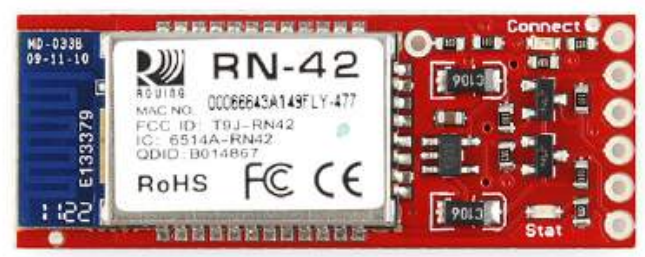

Figure 4.53 - Bluetooth Mate Silver

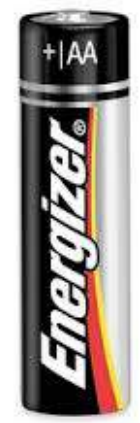

Figure 4.54

Standard AA battery

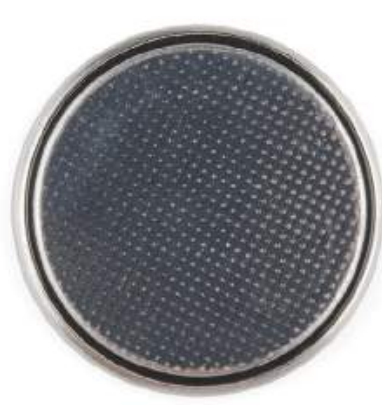

Figure 4.55

Coin cell battery

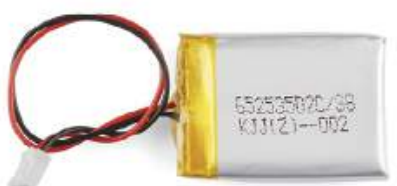

Figure 4.56

LiPo battery 



Once the wrap band was developed the next step was to see how it could be used as a game controller. Three design experiments were conducted, focusing on:

1. Integration with a pre-existing game

2. Use as an exercise assessment tool

3. Developing a specific game with the wrap band and WBA in mind 
Integrating the wrap band with a pre-existing game was the first design experiment. This decision was made because it would show if the hardware could be used successfully as a natural game controller without the focus being on game design and development. The game chosen for initial testing was a 3D iPhone/iPad Operating System (iOS) game called Garglyder (Steel et al., 2013). This game was chosen because the files were easily accessible in order to add a new controller scheme and it was built using Unity, which was the same game engine which previous test with the wrap band have been conducted in.

Using the wrap band in an exercise assessment tool was chosen as the next design experiment. Connecting the wrap band with a tool of this nature would demonstrate if it was possible for the hardware to track exercise movement. The exercise assessment tool used in this design experiment will use the wrap band and a Kinect. The Kinect will focused on the overall body movement and the wrap band will focused on the weight distribution in each foot. This something the Kinect is unable to do.

The final design experiment will involved creating a game that integrates exercise-based movements. The movement will focus on controlled weight distribution between each foot and the various areas of the feet. These sorts of movements will be used because they take advantage of the of the wrap band's pressure sensing capabilities. Controlled weight distribution with visual cues has been shown to help with WBA (McGough et al., 2012). The game will not directly focus on treating WBA. Instead it will focus on a game play that requires the player to alternate and balance their weight on each foot. 

The next exercise was the star excursion test. With this exercise the user would plant one foot and then use the other foot to reach out in a circle touching eight different points. For this exercise to be the successful the following criteria has to be met:

1. Base foot remains firmly planted

2. Equal weight distribution on base foot

3. Light tap on the ball of moving foot

4. Moving foot reaching the eight different angles.

The Kinect was able to measure the base foot position and the angle of the moving foot. To measure if the base foot was remaining firmly planted and the tap of the moving foot the wrap band was used. The pressure on the base foot had to remain evenly distributed throughout the movement of the other foot. If the pressure was not evenly placed on the four sensors during the movement this was considered an unsuccessful test and the program would notify the user with visual cues. The distance the foot travelled was measured from the base foot to the point where the tap happened. Once the tap happened the foot would have to move back next to the base foot. This processes was repeated for the eight points in the star on each foot.

The way the wrap band was used in this design experiment showed how well it could be used as a natural controller. The movements of the controller directly related to what was happening in the software with the aid of the Kinect. In this context the wrap band was just being used as a controller for an assessment tool and not a game. 


\subsection{Custom Game For Hardware}

In previous design experiments it was demonstrated that the wrap band could be used as a game controller with an existing game and as controller for an exercise based assessment tool. The next step was to create a game specific to the hardware with actions relating to exercises. The movements used focused on those related to measuring and treating WBA. This would be done by informing the user of their weight distribution through visual cues which has been shown to improve WBA. The game controls focus on getting the user to alternate and balance their weight in their feet. How the game is controlled will be based on the area in which the user is placing the most weight focusing on the following areas:
1. Balls of feet (Figure 5.3)
2. Heels of feet (Figure 5.4)
3. Left foot (Figure 5.5)
4. Right foot (Figure 5.6)

Before the game was created the game genre needed to be decided. Through the previous design experiment it was shown that the wrap band successfully integrated with the game Garglyder. Garglyder falls into the running platformer game genre. A running platformer is considered a game in which an avatar continuously moves forwards through a procedural generated environment.

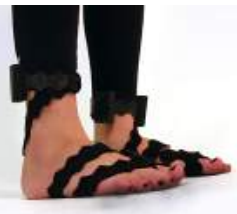

Figure 5.3 - Pressure on the

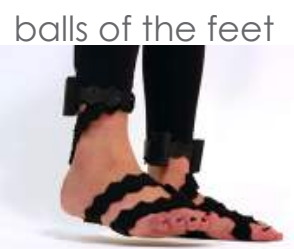

Figure 5.5 - Pressure on the left foot

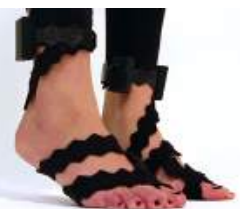

Figure 5.4 - Pressure on the

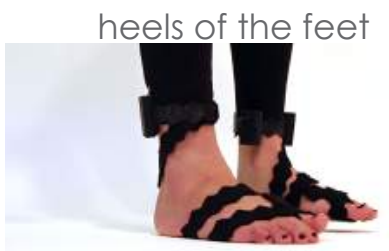

Figure 5.6 - Pressure on the right foot 


\subsubsection{Initial Game Development}

The first step in designing the game was to determine in game movements that could be considered natural in a running platformer game based on the foot controls. To start with abstract shapes were used to focus on controls and movements without having to worry about a cohesive narrative for the game. In the initial iteration of the game the foot controls were applied to a cuboid. When determining what action would control the in game movement three things needed to be considered:

1. Which movements are the most natural

2. Which movements best fit the chosen game genre

3. Which movements are most suited for WBA

Figures 5.7 - 5.16 show and describes how various the actions could be used to control movement, how natural the movement is and how well in fits the chosen game genre.

Using the controls to move the cuboid forward, back, left and right would be appropriate for the game genre but is not a natural control. The user has to lean in order to control the game, and it would make more sense to use the rotational movements to make the cuboid lean. Using the left and right foot controls to rotate around the $y$-axis would be best for the game play however for this to be a natural control the user you have to rotate their body away for the screen. Rotating on the x-axis would be more natural because as the user leans to one side so would the cuboid. After looking at the various controls and possible movements it was decided that the cuboid was not the appropriate shape for the game. 






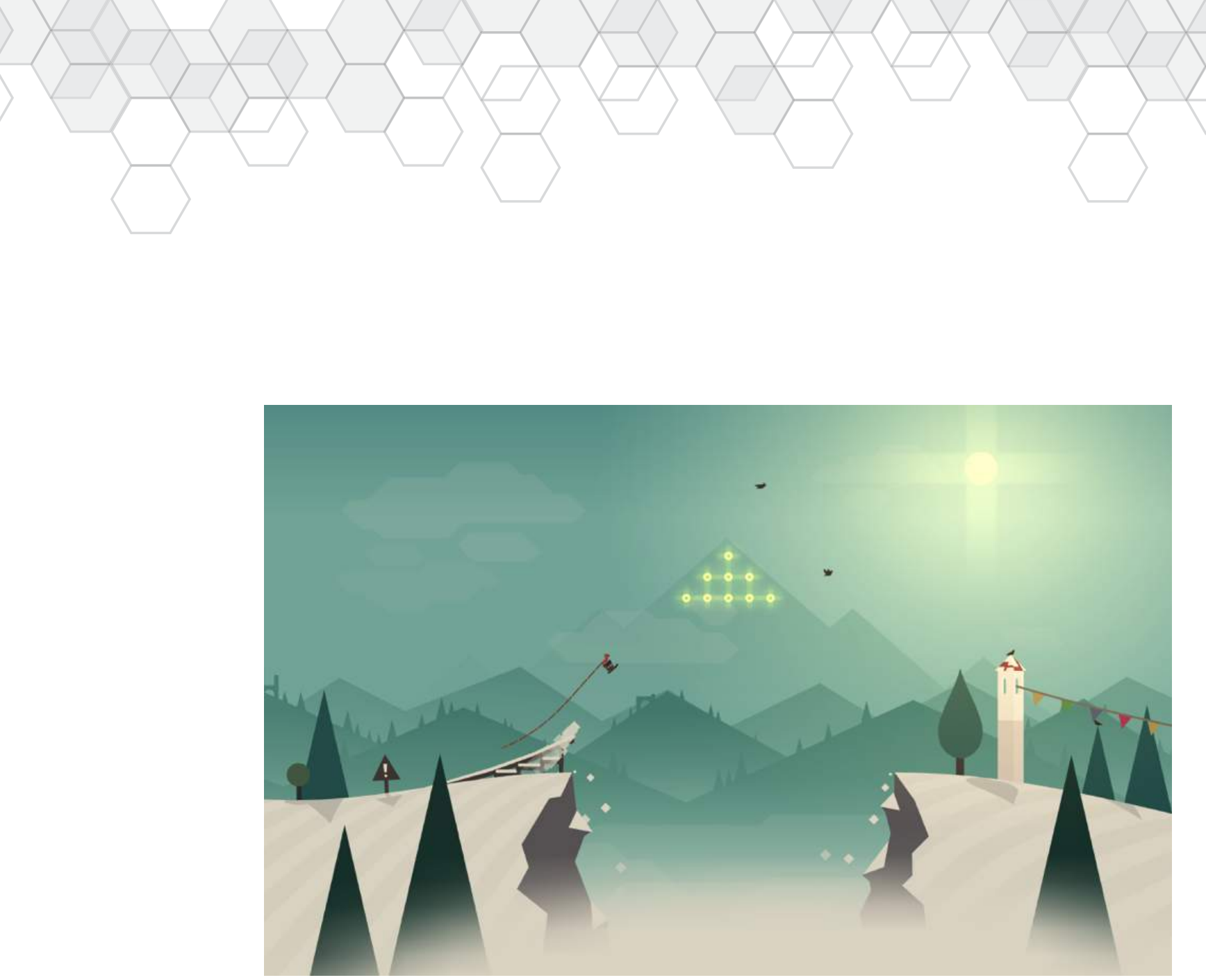

Figure 5.22 - Alto's Adventure (Snowman, 2015)

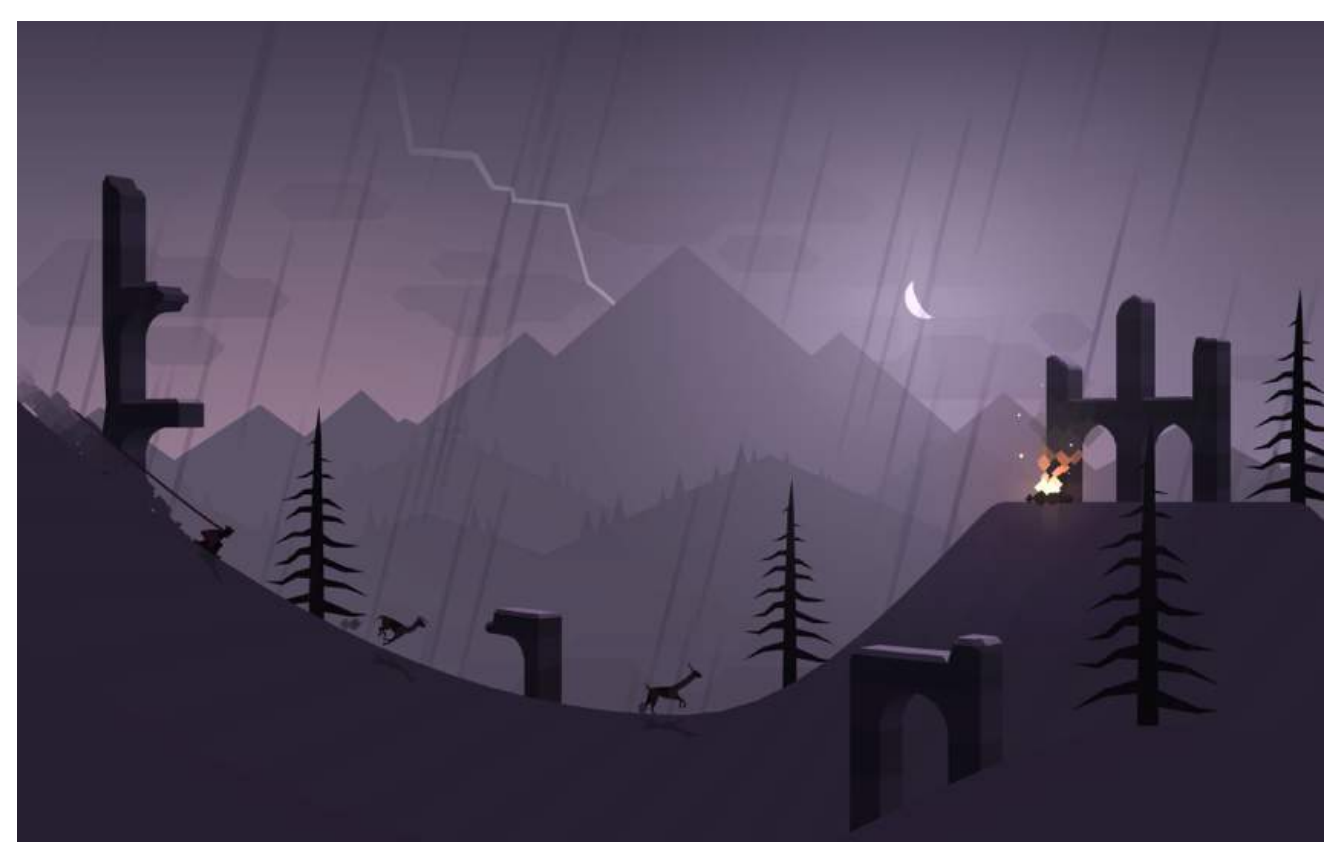

Figure 5.23 - Alto's Adventure (Snowman, 2015) 



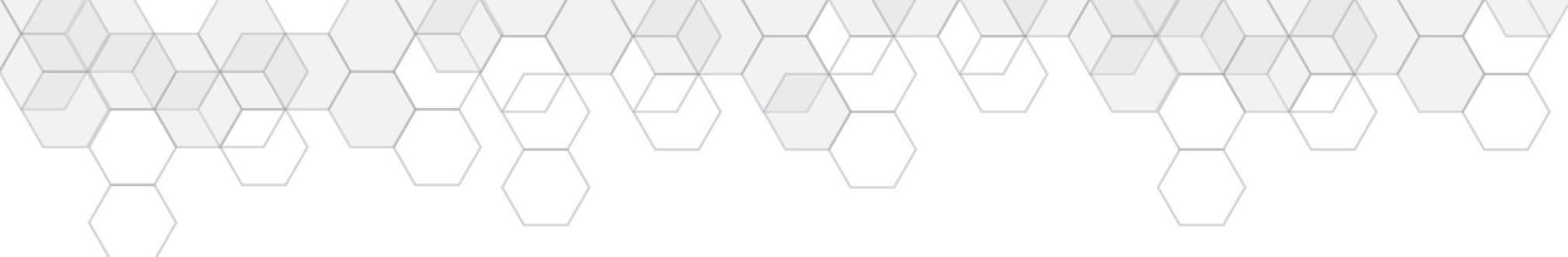

\subsection{Integration with Hardware}

The final step in the creation of the game was to fully integrate it with the hardware. During the creation process the game was tested to see if the sphere could be controlled through the track. These tests were successful but alterations to the code would need to be made based on how well the wrap could be used. The two main things being tested were speed and sensitivity of control. The two wrap bands were worn and then the game was played. The code was then altered based on how well the wrap band controlled the game to ensure the effort put into moving the sphere matched the movement occurring. Once the hardware was integrated with the game the criteria were revisited to determine if the integration had been successful. Final screen shots of the game can be seen in Figures 5.25 -5.27

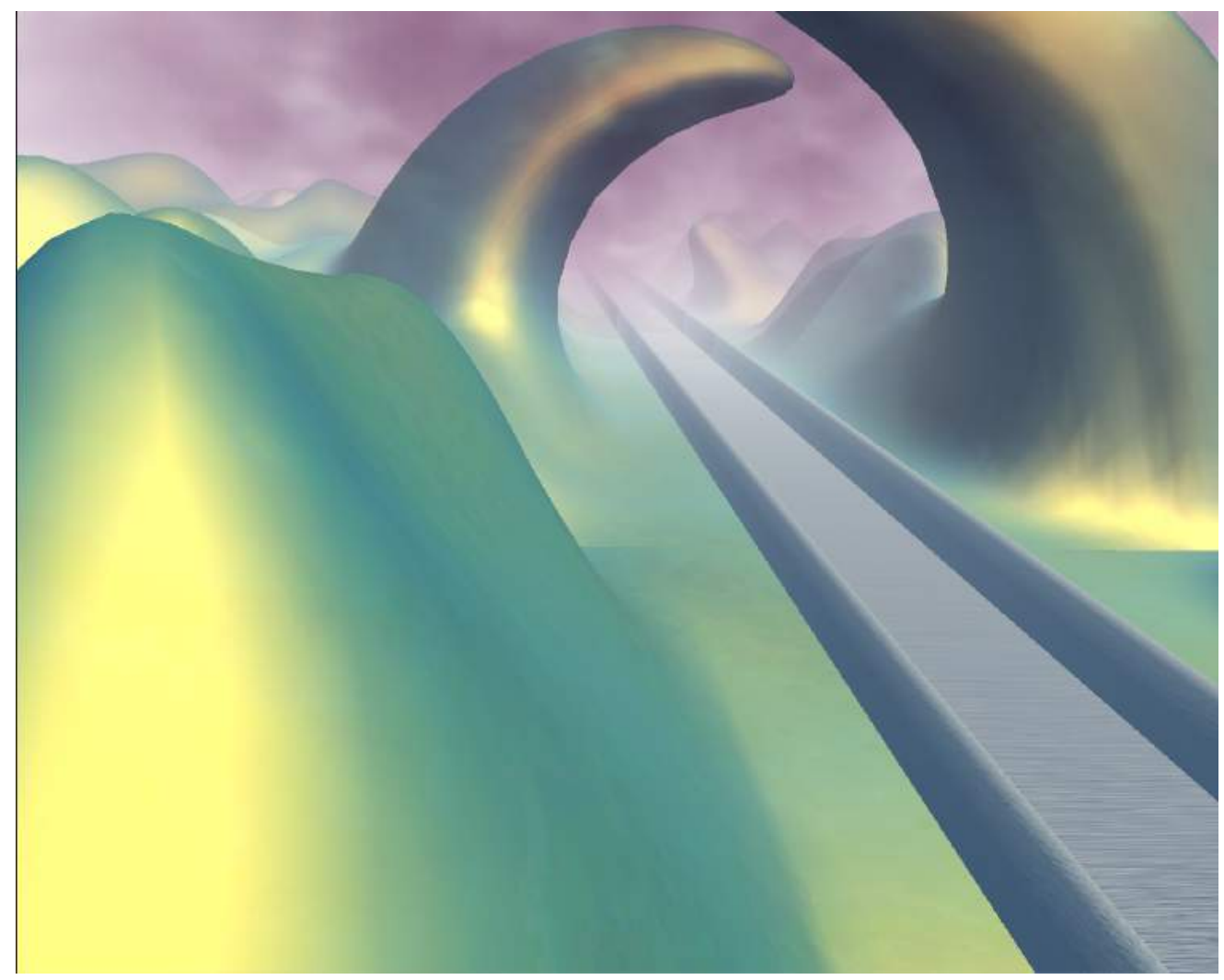

Figure 5.25 


The design process was split into two parts, hardware and software. The hardware part of the project began by focusing on the technical requirements of the wearable to ensure it was capable of providing the required data. This was done using a quantitative evaluation of velostat sensor properties followed by a series of design experiments. These design experiments involved the creation of a wearable taking into consideration manufacturing, aesthetics, durability and cost. This experimentation process resulted in a pressure sensitive wrap band with detachable electronic casing (Figure 6.1)

The next part of the project involved creating software for the wrap band to interact with. This consisted of three parts:

1. Integration with a pre-existing game

2. Use as an exercise assessment tool

3. Developing a specific game with the wrap band and WBA exercises

Integration with a pre-existing game was conducted to ensure the hardware could successfully be used as a game controller. Using the wrap band as an exercise assessment tool showed it could be used to track body movements based on foot pressure. The final experiment involved creating a game to address WBA. This was done by design a game which provided the user with visual cues of how they distributed foot pressure based on the movements of the in-game character.

The hardware created was able to interact with the game as a natural game controller and uses movements which address WBA. While the hardware and software produced during this project have successfully addressed the thesis question, further testing could usefully be done. Further quantitative and qualitative studies would need to be conducted to determine if the system is an appropriate way to treat WBA. The results would then need to be compared to current WBA treatment options before make claims could be made about its effectiveness. 
7. Discussion 
Throughout this thesis it was discovered that a wearable can successfully work as a natural game controller to help with a lower limb injury rehabilitation. While further testing needs to be done to compare the system with current treatment options this thesis does provide a starting point for how wearable technology can be used. These uses could range from pure entertainment to serious gaming in areas such as injury prevention and rehabilitation.

With the emergence of new technologies such as the Oculus Rift, a virtual reality headset, people are looking for ways to make gaming more immersive. A wearable allows a person to move around in a space and use their own body movement or weight to control a game. To make the wearable more effective adding positional sensors to make the wearable aware of its position in a space would allow users to move around freely in the real world to control something in a virtual world.

In terms of injury prevention the wearable could be used while doing sport or exercise. This would provide the user information on how they are performing. Exercising incorrectly for long periods of time can lead to injuries. Having a device which warns people when they first start performing an exercise wrong could potentially decrease their chance of injury. Then if an injury does occur the wearable could help with recovery by providing a measurement of what is going on in the user's body.

Although this technology is still in its infancy and there is clearly a wide range of potential uses. This thesis has detailed one potential use for this technology and its applications. The wrap band created integrates with a custom game in a unique way using gaming for more than just fun. 
References 
Alcorn, A. (1972). PONG. Atari.

Atari. (1974). Gran Trak 10. Atari.

Bateni, H. (2012). Changes in balance in older adults based on use of physical therapy vs the Wii Fit gaming system: a preliminary study. Physiotherapy, 98(3), 211-216. http://doi.org/10.1016/j.physio.2011.02.004

Bristow, S. D. (1977). The History of Video Games. IEEE Transactions on Consumer Electronics, CE-23(1), 58-68. http://doi.org/10.1109/

TCE.1977.266872

Clark, R. A., Bryant, A. L., Pua, Y., McCrory, P., Bennell, K., \& Hunt, M. (2010). Validity and reliability of the Nintendo Wii Balance Board for assessment of standing balance. Gait \& Posture, 31 (3), 307-310. http://doi.org/10.1016/j. gaitpost.2009.11.012

Clark, R. A., Howells, B., Feller, J., Whitehead, T., \& Webster, K. E. (2014). Clinicbased assessment of weight-bearing asymmetry during squatting in people with anterior cruciate ligament reconstruction using Nintendo Wii Balance Boards. Archives of Physical Medicine and Rehabilitation, 95(6), $1156-1161$. http://doi.org/10.1016/j.apmr.2014.02.024

Cook, G., Burton, L., \& Hoogenboom, B. (2006). Pre-Participation Screening: The Use of Fundamental Movements as an Assessment of Function - Part 1. North American Journal of Sports Physical Therapy: NAJSPT, 1 (2), 62-72.

Creswell, J. W. (2011). Research Design: Qualitative, Quantitative, and Mixed Methods Approaches. SAGE Publications.

Crotty, M. (1998). The Foundations of Social Research: Meaning and Perspective in the Research Process. SAGE Publications.

Foo, J., Paterson, K., Williams, G., \& Clark, R. (2013). Low-cost evaluation and real-time feedback of static and dynamic weight bearing asymmetry in patients undergoing in-patient physiotherapy rehabilitation for neurological conditions. Journal of NeuroEngineering and Rehabilitation, 10(1), 74. http:// doi.org/10.1186/1743-0003-10-74

Goldstein, J., LaraOosterbroek, MarkMichielsen, MoniekVan Houten, OscarSalverda, Femke. (1997). Video Games and the Elderly. Social Behavior \& Personality: An International Journal, 25(4), 345. 
Interlink Electronics. (n.d.). Fsr force sensing resistor intergration guide and evaluation parts catalog - 400 series evaluation parts with suggested electrical interfaces. Retrieved from https://www. sparkfun.com/datasheets/ Sensors/Pressure/fsrguide.pdf.

Jonsson, E., Henriksson, M., \& Hirschfeld, H. (2007). Age-related differences in postural adjustments in connection with different tasks involving weight transfer while standing. Gait \& Posture, 26(4), 508-515. http://doi. org/10.1016/j.gaitpost.2006.11.206

Kahlbaugh, P. E., Sperandio, A. J., Carlson, A. L., \& Hauselt, J. (2011). Effects of Playing Wii on Well-Being in the Elderly: Physical Activity, Loneliness, and Mood. Activities, Adaptation \& Aging, 35(4), 331-344. http://doi.org/10.1080/0 1924788.2011 .625218

Kalantari, M., Dargahi, J., Kövecses, J., Mardasi, M. G., \& Nouri, S. (2012). A New Approach for Modeling Piezoresistive Force Sensors Based on Semiconductive Polymer Composites. IEEE/ASME Transactions on Mechatronics, 17(3), 572-581. http://doi.org/10.1109/TMECH.2011.2108664 Khaled, R., Barr, P., Biddle, R., Fischer, R., \& Noble, J. (2009). Game Design Strategies for Collectivist Persuasion. In Proceedings of the 2009 ACM SIGGRAPH Symposium on Video Games (pp. 31-38). New York, NY, USA: ACM. http://doi.org/10.1145/1581073.1581078 Konami. (1998). Dance Dance Revolution. Konami. Kumar, R. (2014). Research Methodology: A Step-by-Step Guide for Beginners. SAGE.

McGough, R., Paterson, K., Bradshaw, E. J., Bryant, A. L., \& Clark, R. A. (2012). Improving Lower Limb Weight Distribution Asymmetry During the Squat Using Nintendo Wii Balance Boards and Real-Time Feedback. Journal of Strength, 26(1), 47-52. http://doi.org/10.1519/JSC.0b013e318220b515 Microsoft. (2013). XBox One. Miyamoto, S. (1981). Donkey Kong. Nintendo. Newhouse, A. (2014, July 9). PS4, PS3, and Vita Combined Have Sold Over 100 Million Systems, Sony Says. Retrieved March 6, 2015, from http://www. gamespot.com/articles/ps4-ps3-and-vita-combined-have-sold-over-100milli/1100-6421018/ 
Nishikado, T. (1978). Space Invaders. Taito.

Perner-Wilson, H., \& Buechley, L. (2010). Making Textile Sensors from Scratch. In Proceedings of the Fourth International Conference on Tangible, Embedded, and Embodied Interaction (pp. 349-352). New York, NY, USA: ACM. http://doi. org/10.1145/1709886.1709972

Playdead, \& Double Eleven. (2010). Limbo.

Rotberg, E., Rubin, O., \& Roger, H. (1980). Battlezone. Atari.

Roy, A. K., Soni, Y., \& Dubey, S. (2013). Enhancing effectiveness of motor rehabilitation using kinect motion sensing technology. In 2013 IEEE Global Humanitarian Technology Conference: South Asia Satellite (GHTC-SAS) (pp. 298-304). http://doi.org/10.1109/GHTC-SAS.2013.6629934

Russell, S., \& Graetz, J. M. (1962). Spacewar.

Skalski, P., Tamborini, R., Shelton, A., Buncher, M., \& Lindmark, P. (2011). Mapping the road to fun: Natural video game controllers, presence, and game enjoyment. New Media \& Society, 13(2), 224-242. http://doi. org/10.1177/1461444810370949 Snowman. (2015). Alto's Adventure. Snowman. Sony Computer Entertainment. (2013). PlayStation 4.

Steel, E., Hoffman, N., Warner, R., Tan, S., Sullivan, T., \& Thornton, G. (2013). Garglyder.

Tanaka, K., Parker, J. R., Baradoy, G., Sheehan, D., Holash, J. R., \& Katz, L. (2012). A Comparison of Exergaming Interfaces for Use in Rehabilitation Programs and Research. Loading..., 6(9). Retrieved from http://journals.sfu. ca/loading/index.php/loading/article/view/107 Thatgamecompany, \& Tricky Pixels. (2012). Journey. Wolf, M. J. P. (Ed.). (2008). The video game explosion: a history from PONG to Playstation and beyond. Westport, Conn: Greenwood Press. 
Appendix 


\section{Appendix}

\section{Sensors}

\subsection{Force Sensing Resistors}

The first sensors this project looked at were force-sensing resistors. These sensors have a high cost per a unit and are not customisable. A further problem with these sensors is that they are not able to endure repeated shear forces (Interlink Electronics, n.d.), a scenario which could potentially occur in our proposed use.

\subsection{Piezoelectric Based Sensing}

This type of sensing would involve the implementation of a grid of piezoelectric crystals. While these would be easy to source, durable and customisable they have the disadvantage of being hard and unable to flex. They need to be implemented in a wearable therefore the ability to be flexible is important. The crystals would cause discomfort to the user and potentially limit their movements.

\subsection{Velostat Based Pressure Sensors}

These sensors have the advantage of being cost effective and easily customisable. They can be made from a range of fabrics making it easy to source the materials and integrate them into a wearable. There is limited literature surrounding exactly how they behave due to the open source and customisable nature of these sensors. These sensors best align with the criteria set out but individual testing would need to be done to determine their suitability prior to implementation. 


\section{Sensor Testing}

\subsection{Base Resistance Testing}

In previous experiments the sensitivity of the sensors and the base resistance affected the range of the measurements. To ensure the sensors were providing the best data a test was designed to identify the base resistance effect. Using $1 \mathrm{~mm}$ thick neoprene a sensor with an area of $625 \mathrm{~mm}^{2}(25 \mathrm{~mm} \times 25 \mathrm{~mm}$ square) was constructed using the process previously stated. The sensor was then placed in a voltage dividing circuit.

The base resistance started at $50 \mathrm{ohm}$ and ranged up to 1 Mohm. The sensor was timed for one minute and the output data recorded for each test. As the base resistance increased so did the variance in the sensors output. These resulted showed the sensor output was more consistent with a lower the base resistance. A base resistance smaller than $50 \mathrm{ohm}$ was also tested however this produced a near zero reading when a weight of $1 \mathrm{Kg}$ was placed on the sensor and would not be useful for the required application.

\subsection{Settling Time Evaluation}

During the base resistance tests it was noted the sensor value began to drift past the initial one minute testing time. To see if the sensors eventually settled on a certain value an experiment was design to see how the sensors behaved over time. To evaluate this a range of weights between $50 \mathrm{~g}$ and $5050 \mathrm{~g}$ were place on the sensors to determine the initial sensor response, evaluate the sensor settling time and see how this could affect the use of the sensors later on. The tests ranged between one minute and one hour and it was shown that the sensors did experience an extended settling time. This was confirmed by external studies using similar sensors (Kalantari, Dargahi, Kövecses, Mardasi, \& Nouri, 2012). Based on this information the sensors would be most useful in devices requiring an on/off input or for evaluating relative changes in force instead of independent absolute values. 


\subsection{Area Dependency}

During experiments sensors with areas of $625 \mathrm{~mm}^{2}(25 \mathrm{~mm} \times 25 \mathrm{~mm}), 2500 \mathrm{~mm}^{2}$ $(50 \mathrm{~mm} \times 50 \mathrm{~mm})$ and $10000 \mathrm{~mm}^{2}(100 \mathrm{~mm} \times 100 \mathrm{~mm})$ were used. It was found the velostat area of did not greatly affect the output of the sensor. What did affect the output was the area in which the weight was being applied. Pressure is an area dependent variable affect by force ( ) and it's the pressure which determines the sensor output (measured in resistance). For example if a $500 \mathrm{~g}$ mass with an area of $1600 \mathrm{~mm}^{2}$ was applied to a sensor with an area of $2500 \mathrm{~mm}^{2}$ and one with an area of $10000 \mathrm{~mm}^{2}$ the pressure (3062.5Pa) experienced by each sensor would be the same. Due to this finding a sensor with a small area such as the $625 \mathrm{~mm}^{2}$ sensor would be most useful for the intended purpose. In the context of using them in a wearable such as a foot the contact area for each sensor will be the same and measuring the relative force between each contact point will be easier to determine. For example you would be able to tell if someone was placing all of his or her mass on a sensor in the heel of the foot because it would be exerting a larger amount of force on the same amount of area as in the ball e.g. For a $60 \mathrm{~kg}$ person placing most of their weight in their heel (approx 59kg) while standing still (gravity is the only acceleration) $\mathrm{n}$ theory smaller sensor would be better but this would create manufacturing issues and for our intended purpose is unnecessary.

\subsection{Fabric Dependency}

The final variable to consider was the thickness of the neoprene used to cover the fabric. The initial assumption was that thicker neoprene would resist compression more and reduce the sensitivity of the sensor. To test the two sensors $100 \mathrm{~mm} \times 100 \mathrm{~mm}$ were constructed one with a thickness of $3 \mathrm{~mm}$ and the other with a thickness of $5 \mathrm{~mm}$. The sensors were then tested with various weights to see how the different thicknesses would react. Testing showed the thicker neoprene did reduce the sensitivity of the sensor. Reduced sensitivity would not be ideal for the intended purpose so thinner fabric would be better. Thinner fabric would also offer the advantage of being less restrictive for the user. Based a small series of tests it appears there is no limit to how thin the fabric can be. 


\section{Use of the CNC Sewing Machine}

\subsection{How the machine works}

The sewing machine used for this project is a Janome Memory Craft 9900. It has a standard sewing mode that provides all the functions of a generic home sewing machine and also has an embroidery mode. The embroidery modes provide the opportunity to sew 2D images in a range of colours. It come with a series of premade designs and also give users the opportunity to create their own. To create design for the machine one option is to create an image in Adobe lllustrators and save it as a jpeg, the jpeg can then be imported into the sewing machine softer and converted to a stitching file. Once the image has been converted to stitches it can be sewn as is or the user can edit the stitching pattern and stitch type. When sewing the machine uses a top stitch and a bobbin stitch which can uses different types of thread depending on the desired outcome.

\subsection{Conductive Thread as a Top Stitch}

Conductive thread is one of the base materials used in creating a pressure sensor. It was important to find out if the CNC sewing machine could sew using this type of thread. With previous sewing machines it was possible to sew using conductive thread but only if the thread was used in the bobbin. A short test was done using the standard sewing mode to see if it was possible to use conductive thread as a top stitch. After the experiments it was discovered that top-stitching could be done and was more successful using specific settings. For best results the stitch length had to be set to greater than $3 \mathrm{~mm}$ the tension had to be on the lowest possible setting and the speed could not be over 400 stitches per minute (spm). It was also discovered that the conductive thread would break due to imperfection in the spool. This was due to the quality of the thread and was hard to avoid however could be rectified by re-threading the machine. 



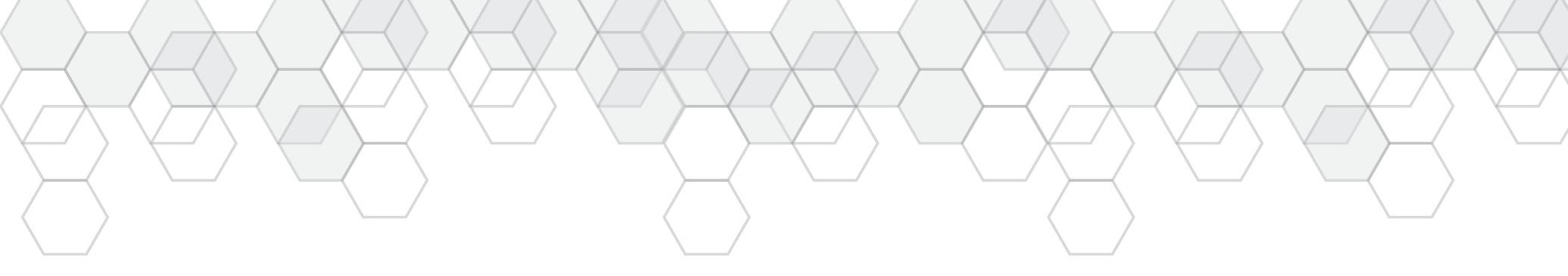

